\title{
ROBUST NUMERICAL METHODS FOR NONLOCAL (AND LOCAL) EQUATIONS OF POROUS MEDIUM TYPE. PART II: SCHEMES AND EXPERIMENTS
}

\author{
FÉLIX DEL TESO, JøRGEN ENDAL, AND ESPEN R. JAKOBSEN
}

ABSTRACT. We develop a unified and easy to use framework to study robust fully discrete numerical methods for nonlinear degenerate diffusion equations

$$
\partial_{t} u-\mathfrak{L}[\varphi(u)]=f(x, t) \quad \text { in } \quad \mathbb{R}^{N} \times(0, T),
$$

where $\mathfrak{L}$ is a general symmetric Lévy type diffusion operator. Included are both local and nonlocal problems with e.g. $\mathfrak{L}=\Delta$ or $\mathfrak{L}=-(-\Delta)^{\frac{\alpha}{2}}, \alpha \in(0,2)$, and porous medium, fast diffusion, and Stefan type nonlinearities $\varphi$. By robust methods we mean that they converge even for nonsmooth solutions and under very weak assumptions on the data. We show that they are $L^{p}$-stable for $p \in[1, \infty]$, compact, and convergent in $C\left([0, T] ; L_{\mathrm{loc}}^{p}\left(\mathbb{R}^{N}\right)\right)$ for $p \in[1, \infty)$. The first part of this project is given in [36] and contains the unified and easy to use theoretical framework. This paper is devoted to schemes and testing. We study many different problems and many different concrete discretizations, proving that the results of [36] apply and testing the schemes numerically. Our examples include fractional diffusions of different orders, and Stefan problems, porous medium, and fast diffusion nonlinearities. Most of the convergence results and many schemes are completely new for nonlocal versions of the equation, including results on high order methods, the powers of the discrete Laplacian method, and discretizations of fast diffusions. Some of the results and schemes are new even for linear and local problems.

\section{Contents}

1. Introduction

2. Preliminaries

3. Numerical schemes - general theory

3.1. The numerical method

3.2. The discretizations $\mathcal{L}^{h}$ and $\varphi^{h}$

3.3. CFL condition for the explicit part

3.4. Comparison, stability, and convergence of the method

3.5. Some extensions

4. Numerical schemes - discretizations - -

4.1. Lagrange interpolation 11

4.2. Discretizations of the local operator $L^{\sigma} \quad 11$

4.3. Discretizations of the singular nonlocal operator $\mathcal{L}_{r}^{\mu} \quad 13$

4.4. Discretizations of the bounded nonlocal operator $\mathcal{L}^{\mu, r} \quad 15$

4.5. A second order discretization of the fractional Laplacian 20

4.6. Approximation of the nonlinearity 22

5. Numerical experiments in 1D 22

5.1. Fractional Heat Equation (explicit scheme) 23

2010 Mathematics Subject Classification. 35A02, 35B30, 35K65, 35D30, 35K65, 35R09, 35R11, 65R20, $76 \mathrm{~S} 05$.

Key words and phrases. Fully discrete, numerical schemes, convergence, uniqueness, distributional solutions, nonlinear degenerate diffusion, porous medium equation, fast diffusion equation, Stefan problem, fractional Laplacian, Laplacian, nonlocal operators, existence, a priori estimates. 
5.2. Fractional Porous Medium Equation (explicit scheme) 24

5.3. Stefan type problem (explicit scheme) 26

5.4. Fractional Fast Diffusion Equation 26

5.5. On the truncation of the domain 28

6. Numerical experiments in 2D 29

Acknowledgements $\quad 31$

$\begin{array}{ll}\text { References } & 32\end{array}$

\section{INTRODUCTION}

We develop a unified and easy to use framework for fully discrete monotone numerical methods of finite difference type for a large class of possibly degenerate nonlinear diffusion equations of porous medium type:

$$
\begin{aligned}
& \partial_{t} u-\mathfrak{L}^{\sigma, \mu}[\varphi(u)]=f(x, t) \quad \text { in } \quad Q_{T}:=\mathbb{R}^{N} \times(0, T), \\
& u(x, 0)=u_{0}(x) \quad \text { on } \quad \mathbb{R}^{N},
\end{aligned}
$$

where $u$ is the solution, $\varphi$ continuous and nondecreasing, and $T>0$. The diffusion operator $\mathfrak{L}^{\sigma, \mu}$ is given as

$$
\mathfrak{L}^{\sigma, \mu}:=L^{\sigma}+\mathcal{L}^{\mu}
$$

with local and nonlocal (anomalous) parts,

$$
\begin{aligned}
& L^{\sigma}[\psi](x):=\operatorname{tr}\left(\sigma \sigma^{T} D^{2} \psi(x)\right), \\
& \mathcal{L}^{\mu}[\psi](x):=\int_{\mathbb{R}^{N} \backslash\{0\}}\left(\psi(x+z)-\psi(x)-z \cdot D \psi(x) \mathbf{1}_{|z| \leq 1}\right) \mathrm{d} \mu(z),
\end{aligned}
$$

where $\sigma=\left(\sigma_{1}, \ldots, \sigma_{P}\right) \in \mathbb{R}^{N \times P}$ for $P \in \mathbb{N}$ and $\sigma_{i} \in \mathbb{R}^{N}, D$ and $D^{2}$ are the gradient and Hessian, $\mathbf{1}_{|z| \leq 1}$ is a characteristic function, and $\mu$ is a nonnegative symmetric measure.

Remark 1.1. By symmetry of $\mu, \lim _{r \rightarrow 0^{+}} \int_{r<|z| \leq 1} z \mathrm{~d} \mu(z)=0$, and we have an equivalent definition of $\mathcal{L}^{\mu}$ in (1.5) in terms of a principal value integral:

$\mathcal{L}^{\mu}[\psi](x)=\lim _{r \rightarrow 0^{+}} \int_{|z|>r}(\psi(x+z)-\psi(x)) \mathrm{d} \mu(z)=$ P.V. $\int_{|z|>0}(\psi(x+z)-\psi(x)) \mathrm{d} \mu(z)$.

The assumptions we impose on $\mathfrak{L}^{\sigma, \mu}$ and $\varphi$ are so mild that many different problems can be modelled by (1.1): Flow in porous media, nonlinear heat transfer, phase transitions, and population dynamics - see e.g. [66] for local problems and $[70,57,14,67]$ for nonlocal problems. Important examples are strongly degenerate Stefan problems with $\varphi(u)=\max (0, a u-b), a \geq 0$, and the full range of porous medium and fast diffusion equations with $\varphi(u)=u|u|^{m-1}$ for any $m \geq 0$. The class of diffusion operators $\mathfrak{L}^{\sigma, \mu}$ coincides with the generators of the symmetric Lévy processes $[7,64,4]$ and includes e.g. the Laplacian $\Delta$, fractional Laplacians $-(-\Delta)^{\frac{\alpha}{2}}, \alpha \in(0,2)$, relativistic Schrödinger operators $m^{\alpha} I-\left(m^{2} I-\Delta\right)^{\frac{\alpha}{2}}$, and even discretizations of these. Since $\sigma$ and $\mu$ may be degenerate or even identically zero, problem (1.1) can be purely nonlocal, purely local, or a combination. An additional challenge both analytically and numerically is the fact that solutions of (1.1) in general can be very irregular and even discontinuous.

The numerical schemes will be defined on a grid $\left(x_{\beta}, t_{j}\right)$ as follows,

$$
U_{\beta}^{j}=U_{\beta}^{j-1}+\Delta t_{j}\left(\mathcal{L}_{1}^{h}\left[\varphi_{1}^{h}\left(U^{j}\right)\right]_{\beta}+\mathcal{L}_{2}^{h}\left[\varphi_{2}^{h}\left(U^{j-1}\right)\right]_{\beta}+F_{\beta}^{j}\right),
$$


where $U_{\beta}^{j} \approx u\left(x_{\beta}, t_{j}\right), \mathcal{L}_{1}^{h}+\mathcal{L}_{2}^{h} \approx \mathfrak{L}^{\sigma, \mu}, \varphi_{i}^{h} \approx \varphi, F_{\beta}^{j} \approx f\left(x_{\beta}, t_{j}\right)$ and $h$ and $\Delta t_{j}$ are the discretization parameters, and the discrete diffusion operators $\mathcal{L}_{i}^{h}$ have a monotone difference representation

$$
\mathcal{L}_{i}^{h}[\psi](x)=\sum_{\beta \neq 0}\left(\psi\left(x+z_{\beta}\right)-\psi(x)\right) \omega_{i, \beta} \quad \text { for } \quad \omega_{i, \beta} \geq 0 .
$$

As we will see, different choices of $\varphi_{1}^{h}, \varphi_{2}^{h}, \mathcal{L}_{1}^{h}, \mathcal{L}_{2}^{h}$ lead to explicit, implicit, $\theta$ methods, and various explicit-implicit methods. In a simple one dimensional case,

$$
\partial_{t} u=\varphi(u)_{x x}-\left(-\partial_{x}^{2}\right)^{\alpha / 2} \varphi(u),
$$

an example of a discretization in our class is given by

$$
\begin{aligned}
U_{m}^{j}=U_{m}^{j-1} & +\frac{\Delta t}{h^{2}}\left(\varphi\left(U_{m+1}^{j}\right)-2 \varphi\left(U_{m}^{j}\right)+\varphi\left(U_{m-1}^{j}\right)\right) \\
& +\Delta t \sum_{k \neq 0}\left(\varphi\left(U_{m+k}^{j-1}\right)-\varphi\left(U_{m}^{j-1}\right)\right) \int_{\left(k-\frac{1}{2}\right) h}^{\left(k+\frac{1}{2}\right) h} \frac{c_{N, \alpha} \mathrm{d} z}{|z|^{N+\alpha}} .
\end{aligned}
$$

The main result of the first part of this project [36] was a unified, rigorous, and easy to use theoretical framework for these schemes. This novel analysis includes well-posedness, $L^{p}$-stability, equicontinuity, compactness, and $L_{\text {loc }}^{p}$-convergence results. These results are very general since they hold for local and nonlocal, linear and nonlinear, non-degenerate and degenerate, and smooth and nonsmooth problems. An important new idea is to work in a sufficiently general class of solutions of (1.1) that allows for atomic (non-absolutely continuous) measures $\mu$ in the definition of $\mathfrak{L}^{\sigma, \mu}$. Since the discrete operator $\mathcal{L}^{h}$ is a nonlocal operator $\mathcal{L}^{\nu}$ with $\nu:=\sum_{\beta \neq 0}\left(\delta_{z_{\beta}}+\delta_{z_{-\beta}}\right) \omega_{\beta}$, it is in the form of $\mathfrak{L}^{\sigma, \mu}$ and can be analyzed with the same powerful $\mathrm{PDE}$ techniques. This analysis requires recent uniqueness results for (1.1) obtained by the authors in [35, 34] - results for bounded distributional solutions or very weak solutions of (1.1) in the generality needed here. The fact that we can use such a weak notion of solution both simplifies the analysis and makes a global theory for all the different problems and schemes we consider here possible. At this point the reader should note that if (1.1) has more regular (bounded) solutions (weak, strong, mild, or classical), then our results still apply because these solutions will coincide with our (unique) distributional solution.

Schemes that converge in such general circumstances are often said to be robust. Consistent numerical schemes are not robust in general, i.e. they need not always converge, or can even converge to false solutions. Such issues are seen especially in nonlinear, degenerate and/or low regularity problems. Our general results are therefore only possible because we have (i) identified a class of schemes with good properties (including monotonicity) and (ii) developed the new mathematical techniques needed to analyse these schemes in the current generality.

In this paper, which is the second part of this project, we have two main objectives: (1) to give many concrete discretizations that fall into the theoretical framework of the first part [36], and (2) to test and verify numerically a number of these schemes for a wide and representative number of examples of problems of the form (1.1).

The scheme (1.6) is essentially determined as soon as we specify $\mathcal{L}_{i}^{h}$ and $\varphi_{i}^{h}$, the discretizations of $\mathfrak{L}^{\sigma, \mu}$ and $\varphi$. The whole of Section 4 is devoted to such concrete discretizations. We start by splitting the diffusion operator $\mathfrak{L}^{\sigma, \mu}$ into local, singular nonlocal, and bounded nonlocal parts, and then explain how these parts can be discretized separately. For the local part, we consider classical finite difference methods and in this context new semi-Lagrangian methods. For the singular nonlocal part, we analyse the trivial discretization and the adapted vanishing viscosity 
approximation, and finally, for the bounded nonlocal part, we consider quadrature methods obtained from interpolation in two different ways. For the first time we apply the so-called powers of the discrete Laplacian method (when $\mathfrak{L}^{\sigma, \mu}=-(-\Delta)^{\alpha}$ ) to diffusion problems, and we explain how non-Lipschitz (including fast diffusion) nonlinearities $\varphi$ have to be approximated to get good explicit schemes.

In every case we check that the discretizations satisfy the conditions of the theoretical framework of [36], and hence we prove that schemes (1.6) involving these discretization are $L^{p}$-stable for $p \in[1, \infty]$ and $L_{\text {loc }}^{p}$-convergent for $p \in[1, \infty)$. We also compute the local truncation errors, and we explain how to combine the methods to get better than first order methods for problems involving fractional Laplace like operators and very high order methods for bounded nonlocal operators. The powers of the discrete Laplacian method is shown to be an order 2 method regardless of the value of $\alpha \in(0,2)$. Many of these schemes and most of the convergence results are new in this context, sometimes even in the linear case. This is especially the case for nonlocal problems. Some important examples here are:

(i) the first high order methods for nonlinear nonlocal diffusions of porous medium type (but see also [43]),

(ii) the first time the powers of discrete Laplacian method is applied to nonlinear problems, and

(iii) the first numerical methods and simulations for nonlocal problems with nonLipschitz ("fast diffusion") nonlinearities.

We also mention that our results provide a rigorous justification for the numerical simulations of Section 7 in [10] for a nonlocal Stefan problem with discontinuous solutions, see Remark 5.1 for more details.

Numerical tests are presented in Section 5-6. We focus on nonlocal problems since there are much less results for such problems in the literature, especially for porous medium type equations. For simplicity, we take the diffusion operator $\mathfrak{L}^{\sigma, \mu}$ to be the fractional Laplacian $-(-\Delta)^{\frac{\alpha}{2}}$ in Section 5. All the well-known one dimensional special cases of (1.1) are then considered: the linear fractional heat equation, the fractional porous medium equation [31], and fractional equations with fast diffusion and Stefan type nonlinearities [31, 10, 3]. In each problem we test and compare four different numerical schemes for different powers $\alpha$ of the fractional Laplacian. Most test problems are set up to have smooth exact solutions, and the numerical tests confirm the theoretical results, in most cases also including the truncation error bounds and the expected convergence rates.

Note that in the Stefan case, we expect that $\varphi(u) \in C^{\gamma}$ for some $\gamma \in(0,1]$, but this is not enough to ensure that $-(-\Delta)^{\frac{\alpha}{2}}[\varphi(u)]$ exists pointwise when $\alpha \geq \gamma$. If this is the case, the scheme does not converge in $L^{\infty}$, but it still converges in $L^{1}$ with the expected rates given by the local truncation error. See Section 5.3 for the details.

We also produce a Stefan type example where the numerical solution is nondifferentiable. Finally, in Section 6 we test a much more complicated problem in two dimensions: A Stefan problem with degenerate local and nonlocal diffusion and nonsmooth castle like initial data.

To perform the numerical computations mentioned above, we have restricted the scheme to a (large) bounded domain and set the numerical solution equal to zero outside. Convergence of the scheme then requires the size of the computational domain to increase as the grid is refined. We briefly discuss the error introduced by the restriction to a bounded domain in Section 5.5.

Related work. In the local linear case, when $\varphi(u)=u$ and $\mu \equiv 0$ in (1.1), numerical methods and analysis can be found in undergraduate text books. In 
the nonlinear case there is a very large literature so we will focus only on some developments that are more relevant to this paper. For porous medium nonlinearities $\left(\varphi(u)=u|u|^{m-1}\right.$ with $\left.m>1\right)$, there are early results on finite element and finite-difference interface tracking methods in [62] and [38] (see also [59]). There is extensive theory for finite volume schemes, see [48, Section 4] and references therein for equations with locally Lipschitz $\varphi$. For finite element methods there is a number of results, including results for fast diffusions $(m \in(0,1))$, Stefan problems, convergence for strong and weak solutions, discontinuous Galerkin methods, see e.g. [63, 45, 46, 44, 72, 61, 58]. Note that the latter paper considers the general form of (1.1) with $\mathfrak{L}^{\sigma, \mu}=\Delta$ and provides a convergence analysis in $L^{1}$. A number of results on finite difference methods for degenerate convection-diffusion equations also yield results for (1.1) in special cases, see e.g. [47, 13, 55, 54]. In particular the results of $[47,55]$ imply our convergence results for a particular scheme when $\varphi$ is locally Lipschitz, $\mathfrak{L}^{\sigma, \mu}=\Delta$, and solutions have a certain additional BV regularity. Finally, we mention very general results on so-called gradient schemes [40, 41] for doubly or triply degenerate parabolic equations. This class of equations include local porous medium type equations as a special case.

In the nonlocal case, the literature is more recent and not so extensive. For the linear case we refer somewhat arbitrarily to $[26,50,51,60]$ and references therein. Here we also mention [28] and its novel finite element plus semigroup subordination approach to discretizing $\mathfrak{L}^{\sigma, \mu}=-(-\Delta)^{\frac{\alpha}{2}}$. Some early results for nonlocal problems came from finite difference quadrature schemes for Bellman equations and fractional conservation laws, see [53, 17, 8] and [39]. For the latter case discontinuous Galerkin and spectral methods were later studied in [25, 23, 71]. The first results that include nonlinear nonlocal versions of (1.1) was probably given in [22]. There, convergence of finite difference quadrature schemes was proven for a convectiondiffusion equation. This result is extended to more general equations and error estimates in [24] and a higher order discretization in [43]. In some cases our convergence results follow from these results (for two particular schemes, $\sigma=0$, and $\varphi$ locally Lipschitz). However, the analysis there is different and more complicated since it involves entropy solutions and Kružkov doubling of variables arguments.

In the purely parabolic case (1.1), the behaviour of the solutions and the underlying theory is different from the convection-diffusion case (especially so in the nonlocal case, see e.g. [30, 31, 68, 29, 69] and [42, 18, 1, 22, 2, 52]). It is therefore important to develop numerical methods and analysis that are specific for this setting. The first (nonlocal) results in this direction seems to be [33, 37]. These papers are based on the extension method [15], and introduce and analyze finite difference methods for the Fractional Porous Medium Equation. The present work is possibly the first not to use the extension method or the regularity of the solution.

Outline. The next section is a short section where we collect the assumptions and well-posedness results for the porous medium type equation (1.1). In section 3 we formulate the numerical schemes and state the main theoretical results. This is a slightly simplified version of the theoretical framework of part 1 of this project [36]. We also give a couple of new results that will greatly simplify the verification of the assumptions of this framework. The main contributions of this paper are then given in the two sections that follows. In Section 4 we introduce the concrete discretizations and prove rigorously that they fall into our theoretical framework, while in Sections 5-6 we present our numerical simulations for all the well-known special cases of (1.1). 


\section{Preliminaries}

In this section we present the assumptions and well-posedness results for the initial value problem (1.1)-(1.2). In this paper we work in the setting of bounded distributional solutions. This is very convenient for numerical analysis since it leads to an easy to work with convergence theory that applies even to very bad problems. By uniqueness it also applies to situations where solutions are more regular, e.g. classical, strong, weak/energy, or mild solutions.

Following [34] (see also [11, 35]) we use the assumptions:

$\left(\mathrm{A}_{\varphi}\right) \quad \varphi: \mathbb{R} \rightarrow \mathbb{R}$ is nondecreasing and continuous;

$\left(\mathrm{A}_{f}\right) \quad f$ is measurable and $\int_{0}^{T}\|f(\cdot, t)\|_{L^{1}\left(\mathbb{R}^{N}\right)}+\|f(\cdot, t)\|_{L^{\infty}\left(\mathbb{R}^{N}\right)} \mathrm{d} t<\infty$;

$\left(\mathrm{A}_{u_{0}}\right) \quad u_{0} \in L^{1}\left(\mathbb{R}^{N}\right) \cap L^{\infty}\left(\mathbb{R}^{N}\right)$; and

$\left(\mathrm{A}_{\mu}\right) \quad \mu$ is a nonnegative symmetric Radon measure on $\mathbb{R}^{N} \backslash\{0\}$ satisfying

$$
\int_{|z| \leq 1}|z|^{2} \mathrm{~d} \mu(z)+\int_{|z|>1} 1 \mathrm{~d} \mu(z)<\infty .
$$

Sometimes we will need stronger assumptions than $\left(\mathrm{A}_{\varphi}\right)$ and $\left(\mathrm{A}_{\mu}\right)$ :

$\left(\operatorname{Lip}_{\varphi}\right) \quad \varphi: \mathbb{R} \rightarrow \mathbb{R}$ is nondecreasing and locally Lipschitz.

$\left(\mathrm{A}_{\mu_{\alpha}}\right) \quad$ There are constants $\alpha \in(0,2)$ and $C \geq 0$ such that for all $r \in(0,1)$,

$$
\int_{|z|<r}|z|^{k} \mathrm{~d} \mu(z) \leq C r^{k-\alpha}, k=2,3,4, \quad \text { and } \int_{r<|z|<1} \mathrm{~d} \mu(z) \leq C r^{-\alpha} .
$$

$\left(\mathrm{A}_{\nu}\right) \quad \nu$ is a nonnegative symmetric Radon measure satisfying $\nu\left(\mathbb{R}^{N}\right)<\infty$.

Remark 2.1. (a) Without loss of generality, we can assume $\varphi(0)=0$ (replace $\varphi(u)$ by $\varphi(u)-\varphi(0))$, and when $\left(\operatorname{Lip}_{\varphi}\right)$ holds, that $\varphi$ is globally Lipschitz (since $u$ is bounded). In the latter case we let $L_{\varphi}$ denote the Lipschitz constant.

(b) Under assumption $\left(\mathrm{A}_{\mu}\right)$, for any $p \in[1, \infty]$ and any $\psi \in C_{\mathrm{c}}^{\infty}\left(\mathbb{R}^{N}\right)$,

$$
\left\|\mathfrak{L}^{\sigma, \mu}[\psi]\right\|_{L^{p}} \leq c\left\|D^{2} \psi\right\|_{L^{p}}\left(|\sigma|^{2}+\int_{|z| \leq 1}|z|^{2} \mathrm{~d} \mu(z)\right)+2\|\psi\|_{L^{p}} \int_{|z|>1} \mathrm{~d} \mu(z) .
$$

(c) When $\mu$ is absolutely continuous w.r.t. the Lebesgue measure $\mathrm{d} z$, assumption $\left(\mathrm{A}_{\mu_{\alpha}}\right)$ means that $\mathrm{d} \mu(z) \leq \frac{C}{|z|^{N+\alpha}} \mathrm{d} z$ for $|z|<1$. The nonlocal operator $\mathcal{L}^{\mu}$ then typically would be a fractional differential operator of order $\alpha \in(0,2)$ (a pseudo differential operator ), like e.g. the fractional Laplacian $(-\Delta)^{\frac{\alpha}{2}}$.

(d) Assumption $\left(\mathrm{A}_{f}\right)$ is equivalent to requiring $f \in L^{1}\left(0, T ; L^{1}\left(\mathbb{R}^{N}\right) \cap L^{\infty}\left(\mathbb{R}^{N}\right)\right)$, an iterated $L^{p}$-space as in e.g. [6]. Note that $L^{1}\left(0, T ; L^{1}\left(\mathbb{R}^{N}\right)\right)=L^{1}\left(Q_{T}\right)$.

Definition 2.1 (Distributional solution). Let $u_{0} \in L_{\mathrm{loc}}^{1}\left(\mathbb{R}^{N}\right)$ and $f \in L_{\mathrm{loc}}^{1}\left(Q_{T}\right)$. Then $u \in L_{\text {loc }}^{1}\left(Q_{T}\right)$ is a distributional (or very weak) solution of (1.1) if for all $\psi \in C_{\mathrm{c}}^{\infty}\left(\mathbb{R}^{N} \times[0, T)\right), \varphi(u) \mathfrak{L}^{\sigma, \mu}[\psi] \in L^{1}\left(Q_{T}\right)$ and

$$
\int_{0}^{T} \int_{\mathbb{R}^{N}}\left(u \partial_{t} \psi+\varphi(u) \mathfrak{L}^{\sigma, \mu}[\psi]+f \psi\right) \mathrm{d} x \mathrm{~d} t+\int_{\mathbb{R}^{N}} u_{0}(x) \psi(x, 0) \mathrm{d} x=0
$$

By Remark 2.1 (b), $\varphi(u) \mathfrak{L}^{\sigma, \mu}[\psi] \in L^{1}$ if e.g. $u \in L^{\infty}$ and $\varphi$ continuous. Distributional solutions exist and are unique in $L^{1} \cap L^{\infty}$.

Theorem 2.2 (Theorem 2.8 in [35] and Theorem 3.1 [34]). Assume $\left(\mathrm{A}_{\varphi}\right),\left(\mathrm{A}_{f}\right)$, $\left(\mathrm{A}_{u_{0}}\right)$, and $\left(\mathrm{A}_{\mu}\right)$. Then there exists a unique distributional solution $u$ of $(1.1)-(1.2)$ such that

$$
u \in L^{1}\left(Q_{T}\right) \cap L^{\infty}\left(Q_{T}\right) \cap C\left([0, T] ; L_{\text {loc }}^{1}\left(\mathbb{R}^{N}\right)\right) .
$$


Note that by $(2.2)$ and $(2.3), u(x, t) \rightarrow u_{0}(x)$ in $L_{\mathrm{loc}}^{1}\left(\mathbb{R}^{N}\right)$ as $t \rightarrow 0^{+}$.

\section{NuMERICAL SCHEMES - GENERAL THEORY}

We introduce and discuss the class of numerical methods that we consider and state the main results about well-posedness, stability, equicontinuity, compactness, and convergence. The proofs of most results in this section are given in [36].

3.1. The numerical method. Our schemes will be defined on time-space grids, nonuniform in time, but uniform in space for simplicity. Our discrete diffusion operators will then have weights and stencils not depending on the position $x$. Let $h>0$, the cube $R_{h}=h\left(-\frac{1}{2}, \frac{1}{2}\right]^{N}$, and $\mathcal{G}_{h}$ be the uniform spatial grid

$$
\mathcal{G}_{h}:=h \mathbb{Z}^{N}=\left\{x_{\beta}:=h \beta: \beta \in \mathbb{Z}^{N}\right\} .
$$

The nonuniform time grid is

$$
\mathcal{T}_{\Delta t}^{T}=\left\{t_{j}\right\}_{j=0}^{J} \quad \text { for } \quad 0=t_{0}<t_{1}<\ldots<t_{J}=T .
$$

Let $\mathbb{J}:=\{1, \ldots, J\}$, and denote the time steps by

$$
\Delta t_{j}=t_{j}-t_{j-1}, \quad j \in \mathbb{J}, \quad \text { and } \quad \Delta t=\max _{j \in \mathbb{J}} \Delta t_{j}
$$

On the grid $\mathcal{G}_{h} \times \mathcal{T}_{\Delta t}^{T}$ we define a class of numerical approximations of (1.1) by discretizing in time and space using monotone finite difference (quadrature) approximations. Using a $\theta$-method in time, the resulting scheme can be written as

$$
U_{\beta}^{j}=U_{\beta}^{j-1}+\Delta t_{j}\left(\theta \mathcal{L}^{h}\left[\varphi\left(U^{j}\right)\right]_{\beta}+(1-\theta) \mathcal{L}^{h}\left[\varphi^{h}\left(U^{j-1}\right)\right]_{\beta}+F_{\beta}^{j}\right)
$$

for $\beta \in \mathbb{Z}^{N}$ and $j \in \mathbb{J}$ where the discrete diffusion operator $\mathcal{L}^{h}$ is given by

$$
\mathcal{L}^{h}[\psi](x)=\sum_{\beta \neq 0}\left(\psi\left(x+z_{\beta}\right)-\psi(x)\right) \omega_{\beta, h} \quad \text { with } \quad z_{\beta} \in \mathcal{G}_{h} .
$$

We will always assume $z_{\beta}=-z_{-\beta}, \omega_{\beta, h}=\omega_{-\beta, h} \geq 0$, and $\sum_{\beta \neq 0} \omega_{\beta}<+\infty$, see Definition 3.1 and Lemma 3.1 below. Then $\mathcal{L}^{h}$ is a monotone finite difference operator with stencil $\mathcal{S}=\left\{z_{\beta}\right\}_{\beta}$ and weights $\left\{\omega_{\beta, h}\right\}_{\beta}$. Note that the scheme is explicit when $\theta=0$, implicit when $\theta=1$, and Crank-Nicholson like when $\theta=\frac{1}{2}$.

Formally we want $U_{\beta}^{j} \approx u\left(x_{\beta}, t_{j}\right), \mathcal{L}^{h} \approx \mathfrak{L}^{\sigma, \mu}, \varphi^{h} \approx \varphi$ and $F_{\beta}^{j} \approx f\left(x_{\beta}, t_{j}\right)$. For $\mathcal{L}^{h}$ and $\varphi^{h}$ this means that we have to impose consistency assumptions, see Definition 3.1(ii) and Definition 3.2(ii) below. But since $u$ and $f$ need not be continuous and point values are not always defined or useful, we will interpret $U$ and $F$ as piecewise polynomial approximations. In this paper we restrict to piecewise constant approximations defined from cell-averages for simplicity. Hence as initial data for the scheme we take

$$
U_{\beta}^{0}:=\frac{1}{h^{N}} \int_{x_{\beta}+R_{h}} u_{0}(x) \mathrm{d} x, \quad F_{\beta}^{j}:=\frac{1}{h^{N} \Delta t_{j}} \int_{t_{j}-\Delta t_{j}}^{t_{j}} \int_{x_{\beta}+R_{h}} f(x, \tau) \mathrm{d} x \mathrm{~d} \tau .
$$

Of course if $f$ and $u_{0}$ are continuous, we could use $U_{\beta}^{0}:=u_{0}\left(x_{\beta}\right)$ and $F_{\beta}^{j}=f\left(x_{\beta}, t_{j}\right)$ instead and all the results below would remain valid.

3.2. The discretizations $\mathcal{L}^{h}$ and $\varphi^{h}$. An admissible discretization $\mathcal{L}^{h}$ of $\mathfrak{L}$ should be (i) monotone, symmetric, (ii) consistent, and (iii) satisfy some uniform Levy integrability condition (which is trivial in the local case). In the next definition we will use that $\mathcal{L}^{h}=\mathcal{L}^{\nu_{h}}$ where $\mathcal{L}^{\nu_{h}}$ is a Levy operator like $\mathfrak{L}$ defined as

$$
\mathcal{L}^{\nu_{h}}[\psi]:=\int_{|z|>0}(\psi(x+z)-\psi(x)) \mathrm{d} \nu_{h}(z) \quad \text { with } \quad \nu_{h}(z)=\sum_{\beta \neq 0} \delta_{z_{\beta}}(z) \omega_{\beta, h}
$$


This surprising observation along with the sufficiently general well-posedness result in Section 3, are key ingredients that make our theory work.

Definition 3.1. A family $\left\{\mathcal{L}^{h}\right\}_{h>0}$ of discretizations of $\mathfrak{L}$ is admissible if it is

(i) in the class $\left(\mathrm{A}_{\nu}\right): \mathcal{L}^{h}=\mathcal{L}^{\nu_{h}}$ for a measure $\nu_{h}$ satisfying $\left(\mathrm{A}_{\nu}\right)$ for all $h>0$,

(ii) consistent: for every $\psi \in C_{c}^{\infty}\left(\mathbb{R}^{N}\right)$,

$$
\left\|\mathfrak{L}[\psi]-\mathcal{L}^{h}[\psi]\right\|_{L^{1}\left(\mathbb{R}^{N}\right)} \rightarrow 0 \quad \text { as } \quad h \rightarrow 0^{+},
$$

(iii) uniformly in $\left(\mathrm{A}_{\mu}\right)$ :

$$
\sup _{h<1} \sum_{\beta \neq 0}\left(\left|z_{\beta}\right|^{2} \wedge 1\right) \omega_{\beta, h}<+\infty .
$$

Note that $\left\|\mathfrak{L}[\psi]-\mathcal{L}^{h}[\psi]\right\|_{L^{1}\left(\mathbb{R}^{N}\right)}$ is the Local Truncation Error of $\mathcal{L}^{h}$ (in $L^{1}$ ), and that in view of (FD2), condition (UL) can equivalently be written as

$$
\sup _{h<1} \int_{|z|>0}|z|^{2} \wedge 1 \mathrm{~d} \nu_{h}(z)<+\infty .
$$

Lemma 3.1. The operators $\left\{\mathcal{L}^{h}\right\}_{h>0}$ defined in (FD) are in the class $\left(\mathrm{A}_{\nu}\right)$ if and only if $z_{\beta}=-z_{-\beta}, \omega_{\beta, h}=\omega_{-\beta, h} \geq 0$, and $\sum_{\beta \neq 0} \omega_{\beta}<+\infty$.

Proof. Since $\nu_{h}(z)=\sum_{\beta \neq 0} \delta_{z_{\beta}}(z) \omega_{\beta, h}$, equivalence for the symmetry and nonnegativity part of $\left(\mathrm{A}_{\nu}\right)$ follows immediately. Equivalence for the boundedness follows from $\nu_{h}\left(\mathbb{R}^{N}\right)=\sum_{\beta \neq 0} \delta_{z_{\beta}}\left(\mathbb{R}^{N}\right) \omega_{\beta, h}=\sum_{\beta \neq 0} \omega_{\beta, h}$.

Assumption (UL) may seem unusual, but it is in fact very natural in view of $\left(A_{\mu}\right)$. It is trivial to verify for local problems, and we now provide a very easy to use sufficient condition for it to hold in the general case.

Proposition 3.2. Assume $\left(\mathrm{A}_{\mu}\right), \mathfrak{L}$ is defined by $(1.3)-(1.5)$, and $\left\{\mathcal{L}^{\nu_{h}}\right\}_{h>0}$ defined by (FD) is in the class $\left(\mathrm{A}_{\nu}\right)$. Then (UL2) holds if

$$
\mathcal{L}^{\nu_{h}}[\psi](x) \stackrel{h \rightarrow 0^{+}}{\longrightarrow} \mathfrak{L}[\psi](x) \quad \text { for all } \quad x \in \mathbb{R}^{N}, \quad \psi \in C_{\mathrm{c}}^{\infty}\left(\mathbb{R}^{N}\right) .
$$

Remark 3.3. (3.2) follows e.g. from $L^{\infty}$-consistency, $\left\|\mathfrak{L}[\psi]-\mathcal{L}^{\nu_{h}}[\psi]\right\|_{L^{\infty}\left(\mathbb{R}^{N}\right)} \rightarrow 0$ as $h \rightarrow 0^{+}$for all $\psi \in C_{\mathrm{c}}^{\infty}\left(\mathbb{R}^{N}\right)$.

Proof of Proposition 3.2. By (FD2), the Taylor expansion

$$
\psi(x+z)=\psi(x)+z \cdot D \psi(x)+\int_{0}^{1}(1-t) z^{T} D^{2} \psi(x+t z) z \mathrm{~d} t,
$$

and since $\int_{|z|<1} z \mathrm{~d} \nu_{h}(z)=0$ by the symmetry of $\nu_{h}$, we find that

$$
\begin{aligned}
\mathcal{L}^{\nu_{h}}[\psi](x)= & \int_{|z| \leq 1} \int_{0}^{1}(1-t) z^{T} D^{2} \psi(x+t z) z \mathrm{~d} t \mathrm{~d} \nu_{h}(z) \\
& +\int_{|z|>1}(\psi(x+z)-\psi(x)) \mathrm{d} \nu_{h}(z) .
\end{aligned}
$$

Then we take $\psi \in C_{c}^{\infty}$ such that $\psi(x)=-1+|x|^{2}$ for $|x| \leq 1$ and $\psi(x) \geq 0$ for $|x|>1$. Since $|t z|<1$ in the first integral above,

$$
\begin{aligned}
\mathcal{L}^{\nu_{h}}[\psi](0) & =\int_{|z| \leq 1}|z|^{2} \mathrm{~d} \nu_{h}(z)+\int_{|z|>1} \psi(z) \mathrm{d} \nu_{h}(z)-\psi(0) \int_{|z|>1} \mathrm{~d} \nu_{h}(z) \\
& \geq \int_{|z| \leq 1}|z|^{2} \mathrm{~d} \nu_{h}(z)+0+\int_{|z|>1} \mathrm{~d} \nu_{h}(z) \geq \int_{|z|>0}\left(|z|^{2} \wedge 1\right) \mathrm{d} \nu_{h}(z)
\end{aligned}
$$


By $\left(\mathrm{A}_{\mu}\right)$, the bound (2.1) holds, and then by (3.2) we conclude that

$$
\begin{aligned}
\sup _{h<1} \int_{|z|>0}\left(|z|^{2} \wedge 1\right) \mathrm{d} \nu_{h}(z) & \leq \sup _{h<1} \mathcal{L}^{\nu_{h}}[\psi](0) \\
& \leq|\mathfrak{L}[\psi](0)|+\sup _{h<1}\left|\left(\mathcal{L}^{\nu_{h}}-\mathfrak{L}\right)[\psi](0)\right|<+\infty
\end{aligned}
$$

The proof is complete.

In most situations we can simply take the nonlinearity $\varphi_{h}=\varphi$, but sometimes it is useful to approximate also $\varphi$. We will see below that this is true especially for fast diffusions.

Definition 3.2. A family $\left\{\varphi^{h}\right\}_{h>0}$ of approximation of $\varphi$ is admissible if it is

(i) in the class $\left(\operatorname{Lip}_{\varphi}\right)$ for every $h>0, \varphi^{h}$ satisfy $\left(\operatorname{Lip}_{\varphi}\right)$,

(ii) consistent: $\varphi^{h} \rightarrow \varphi$ locally uniformly as $h \rightarrow 0^{+}$.

3.3. CFL condition for the explicit part. A crucial property in our convergence analysis is monotonicity. Our schemes are monotone under the CFL condition:

$$
\Delta t(1-\theta) L_{\varphi^{h}} \nu^{h}\left(\mathbb{R}^{N}\right) \leq 1
$$

where $L_{\varphi^{h}}$ denotes the Lipschitz constant of $\varphi^{h}$. Note that the condition always holds if $\theta=1$ and the scheme is implicit. If the scheme has some explicit part, $\theta \in[0,1)$, then this condition gives a relation between $\Delta t$ and $h$. In the local case, we typically have $\nu_{h}\left(\mathbb{R}^{N}\right)=O\left(h^{-2}\right)$, and the CFL condition becomes the classical

$$
\Delta t \leq C h^{2} \text {. }
$$

In the (explicit and) nonlocal case, typically $\nu_{h}\left(\mathbb{R}^{N}\right)=O\left(h^{-\alpha}\right)$ for some $\alpha \in(0,2)$ $\left(\right.$ e.g $\left.\nu_{h} \sim|z|^{-N-\alpha}\right), \nu_{h}\left(\mathbb{R}^{N}\right)=O(|\log (h)|)\left(\right.$ e.g $\left.\nu_{h} \sim|z|^{-N} e^{-|z|}\right)$ or $\nu_{h}\left(\mathbb{R}^{N}\right)<\tilde{C}$ (e.g $\nu_{h} \sim f \in L^{1}\left(\mathbb{R}^{N}\right)$ ), and the CFL condition becomes

$$
\Delta t \leq C h^{\alpha}, \quad \Delta t \leq C \frac{1}{|\log (h)|} \quad \text { or } \quad \Delta t \leq C .
$$

We refer to [36] for more details and the origin of such conditions.

Remark 3.4. Note that $\varphi^{h}$ has to be Lipschitz for the CFL condition to make sense. Hence if $\varphi$ is not Lipschitz (the fast diffusion case), it must be replaced by a Lipschitz approximation to obtain a monotone explicit scheme. The Lipschitz constant $L_{\varphi^{h}}$ will then blow up as $h \rightarrow 0$, and the overall CFL condition is worse than in the Lipschitz case. See Section 5.4.2 for examples.

3.4. Comparison, stability, and convergence of the method. By [36] our numerical method has the following list of properties.

Theorem 3.5 (Existence and uniqueness). Assume $\left(\mathrm{A}_{f}\right),\left(\mathrm{A}_{u_{0}}\right)$ and $\left(\mathrm{A}_{\varphi}\right), \mathcal{L}^{h}$ defined in (FD) satisfies $\left(\mathrm{A}_{\nu}\right), \varphi^{h}$ is in the class $\left(\operatorname{Lip}_{\varphi}\right)$, and $h, \Delta t>0$ are such that (CFL) holds. Then there exists a unique solution $U_{\alpha}^{j}$ of (3.1) such that

$$
\sum_{j \in \mathbb{J}} \sum_{\beta}\left|U_{\alpha}^{j}\right|<\infty
$$

Theorem 3.6 (Properties and convergence). Assume $\left(\mathrm{A}_{\mu}\right),\left(\mathrm{A}_{\varphi}\right), u_{0}, v_{0}$ satisfy $\left(\mathrm{A}_{u_{0}}\right), f, g$ satisfy $\left(\mathrm{A}_{f}\right),\left\{\mathcal{L}^{h}\right\}_{h>0}$ and $\left\{\varphi^{h}\right\}_{h>0}$ are admissible approximations of $\mathfrak{L}$ and $\varphi, \Delta t=o_{h}(1)$ such that (CFL) holds, and $U_{\beta}^{j}, V_{\beta}^{j}$ are solutions of the scheme (3.1) with data $u_{0}, v_{0}$ and $f, g$. Then

(b) (Monotone) If $U_{\beta}^{0} \leq V_{\beta}^{0}$ and $F_{\beta}^{j} \leq G_{\beta}^{j}$ for all $\beta$, then $U_{\beta}^{j} \leq V_{\beta}^{j}$ for all $\beta, j \geq 0$.

(c) (L1'-stable) $\sum_{\beta}\left|U_{\beta}^{j}\right| \leq \sum_{\beta}\left|U_{\beta}^{0}\right|+\sum_{l=1}^{j} \sum_{\beta}\left|F_{\beta}^{l}\right| \Delta t_{l}$. 
(d) $\left(L^{\infty}\right.$-stable $) \sup _{\beta}\left|U_{\beta}^{j}\right| \leq \sup _{\beta}\left|U_{\beta}^{0}\right|+\sup _{\beta} \sum_{l=1}^{j}\left|F_{\beta}^{l}\right| \Delta t_{l}$.

(e) (Conservative) If $\varphi^{h}$ satisfy $\left(\operatorname{Lip}_{\varphi}\right), \sum_{\beta} U_{\beta}^{j}=\sum_{\beta} U_{\beta}^{0}+\sum_{l=1}^{j} \sum_{\beta} F_{\beta}^{l} \Delta t_{l}$.

(f) $\left(L^{1}\right.$-contractive) $\sum_{\beta}\left(U_{\beta}^{j}-V_{\beta}^{j}\right)^{+} \leq \sum_{\beta}\left(U_{\beta}^{0}-V_{\beta}^{0}\right)^{+}+\sum_{l=1}^{j} \sum_{\beta}\left(F_{\beta}^{l}-G_{\beta}^{l}\right)^{+} \Delta t_{l}$.

(g) (Equicontinuity in time) For all compact sets $K \subset \mathbb{R}^{N}$ there exists a modulus of continuity $\Lambda_{K}$ (independent of $h$ and $\Delta t$ ) such that

$$
h^{N} \sum_{x_{\beta} \in \mathcal{G}_{h} \cap K}\left|U_{\beta}^{j}-U_{\beta}^{j-k}\right| \leq \Lambda_{K}\left(t_{j}-t_{j-k}\right)+|K| \int_{t_{j-k}}^{t_{j}}\|f(\cdot, \tau)\|_{L^{\infty}\left(\mathbb{R}^{N}\right)} \mathrm{d} \tau .
$$

(h) (Convergence) There exists a unique distributional solution $u \in L^{1}\left(Q_{T}\right) \cap$ $L^{\infty}\left(Q_{T}\right) \cap C\left([0, T] ; L_{\mathrm{loc}}^{1}\left(\mathbb{R}^{N}\right)\right)$ of (1.1)-(1.2) and for all compact sets $K \subset \mathbb{R}^{N}$,

$$
\|U-u\|_{K}:=\max _{t_{j} \in \mathcal{T}_{\Delta t}^{T}}\left\{\sum_{x_{\beta} \in \mathcal{G}_{h} \cap K} h^{N}\left|U_{\beta}^{j}-u\left(x_{\beta}, t_{j}\right)\right|\right\} \rightarrow 0 \quad \text { as } h \rightarrow 0^{+} .
$$

Note that our schemes are stable in $L^{p}$ for any $p \in[1, \infty]$ by interpolation. The discrete norm convergence results is equivalent to convergence in $C\left([0, T] ; L_{\mathrm{loc}}^{1}\left(\mathbb{R}^{N}\right)\right)$ for interpolants of the numerical solution (piecewise constant in space and piecewise linear in time), see [36] for the details. Convergence was proved through a compactness argument in this space, where equicontinuity results like Theorem 3.6 (f) and (g) were needed. By the $L^{p}$ stability, convergence also holds in $C\left([0, T] ; L_{\mathrm{loc}}^{p}\left(\mathbb{R}^{N}\right)\right)$ for all $p \in[1, \infty)$.

3.5. Some extensions. As shown in [36], the results of Theorem 3.5 and Theorem 3.6 also hold for a larger class of schemes,

$$
U_{\beta}^{j}=U_{\beta}^{j-1}+\Delta t_{j}\left(\sum_{k=1}^{n} \mathcal{L}_{k}^{h}\left[\varphi_{k}^{h}\left(U^{j}\right)\right]_{\beta}+\sum_{k=n+1}^{m} \mathcal{L}_{k}^{h}\left[\varphi_{k}^{h}\left(U^{j-1}\right)\right]_{\beta}+F_{\beta}^{j}\right),
$$

where $n, m \in \mathbb{N}$ with $n \leq m$ and

$$
\sum_{k=1}^{n} \mathcal{L}_{k}^{h}\left[\varphi_{k}^{h}\left(U_{h}^{j}\right)\right](x)+\sum_{k=n+1}^{m} \mathcal{L}_{k}^{h}\left[\varphi_{k}^{h}\left(U_{h}^{j-1}\right)\right](x) \approx \mathfrak{L}[\varphi(u)]\left(x, t_{j}\right) .
$$

Depending on the choices of $\mathcal{L}_{k}^{h}$ and $\varphi_{k}^{h}$, we can then get many different schemes:

(1) Discretizing separately the different parts of the operator

$$
\mathfrak{L}=\mathfrak{L}^{\sigma, \mu}=L^{\sigma}+\mathcal{L}_{\text {sing }}^{\mu}+\mathcal{L}_{\text {bnd }}^{\mu},
$$

e.g. the local, singular nonlocal, and bounded nonlocal parts, corresponds to different choices for $\mathcal{L}_{k}^{h}$. See Section 4 for a detailed discussion.

(2) Explicit schemes $(\theta=0)$, implicit schemes $(\theta=1)$, or combinations like CrankNicholson $\left(\theta=\frac{1}{2}\right)$, follow by the choices

$$
\mathcal{L}_{1}^{h}=\theta \mathcal{L}^{h} \quad \text { and } \quad \mathcal{L}_{2}^{h}=(1-\theta) \mathcal{L}^{h} .
$$

(3) Combinations of type (1) and (2) schemes, e.g. implicit discretization of the unbounded part of $\mathfrak{L}^{\sigma, \mu}$ and explicit discretization of the bounded part. 


\section{NumericAl SCHEMES - DiscRetizations}

In this section we explore known and find new approximations of $\mathfrak{L}$ and $\varphi$, and in every case we show that they are admissible in the sense of Definitions 3.1 and 3.2 (see also Lemma 3.1) and hence yield monotone, stable, and convergent numerical schemes for (1.1) - (1.2) by Theorem 3.6. Many of these schemes are completely new in this setting, and even for many of the known schemes, the convergence results are new. For the diffusion operator $\mathfrak{L}=\mathfrak{L}^{\sigma, \mu}=\mathcal{L}^{\mu}+L^{\sigma}$, we present a series of possible discretizations of both the nonlocal part $\mathcal{L}^{\mu}$ of the form (1.5) satisfying $\left(\mathrm{A}_{\mu}\right)$ and the local second order elliptic operator $L^{\sigma}$ given by (1.4). Most of these discretizations apply to all such operators and are not restricted to the fractional Laplacian/Laplacian. We end the exposition by discussing how to handle nonLipschitz merely continuous nonlinearities $\varphi$, and hence also fast diffusions.

The nonlocal operator $\mathcal{L}^{\mu}$ has a possibly singular part and a nonsingular part that can (and often should) be discretized separately: for $r>0$,

$$
\begin{aligned}
\mathcal{L}^{\mu}[\psi](x)= & \int_{0<|z| \leq r}(\psi(x+z)-\psi(x)-z \cdot D \psi(x)) \mathrm{d} \mu(z) \\
& +\int_{|z|>r}(\psi(x+z)-\psi(x)) \mathrm{d} \mu(z) \\
= & : \mathcal{L}_{r}^{\mu}[\psi](x)+\mathcal{L}^{\mu, r}[\psi](x) .
\end{aligned}
$$

When we discretize this operator, we have to take $h \leq r=o_{h}(1)$ where $h$ is the discretization in space parameter. Often we can simply take $r=h$, but in some cases a different choice can produce higher order discretizations. We will present admissible discretizations for general measures $\mu$ and state their Local Truncation Error (LTE). We also give the LTE when the nonlocal operator is a fractional derivative in the sense of $\left(\mathrm{A}_{\mu_{\alpha}}\right)$.

4.1. Lagrange interpolation. Let $\left\{p_{\beta}^{k}\right\}_{\beta \in \mathbb{Z}^{N}}$ be the basis of piecewise Lagrange polynomials of order $k$ on the grid $\mathcal{G}_{h}$, i.e. $\sum_{\beta} p_{\beta}^{k}(x) \equiv 1$ for all $x \in \mathbb{R}^{N}$ and $p_{\beta}^{k}\left(z_{\gamma}\right)=1$ for $\beta=\gamma$ and zero otherwise. Since the grid is uniform, we may define these functions in a tensorial way for $N>1$ (direction by direction). On $\mathcal{G}_{h}$ the Lagrange polynomial interpolant of order $k$ of a function $\psi$ is given by

$$
I_{h}^{k}[\psi](z):=\sum_{\beta \neq 0} \psi\left(z_{\beta}\right) p_{\beta}^{k}(z),
$$

and if $\psi \in C_{c}^{\infty}\left(\mathbb{R}^{N}\right)$, the corresponding interpolation error is

$$
\left\|I_{h}^{k}[\psi]-\psi\right\|_{L^{p}\left(\mathbb{R}^{N}\right)}=C\left\|D^{k+1} \psi\right\|_{L^{p}\left(\mathbb{R}^{N}\right)} h^{k+1}
$$

where $C=C(k, p)$ and $p=\{1, \infty\}$ (cf. e.g. [19]). Since the grid is uniform, the $p_{\beta}^{k}$-basis will have a lot of symmetries. E.g. when $k=1$ (linear interpolation), $0 \leq p_{\beta}^{1}(x)=p_{0}^{1}\left(x-z_{\beta}\right)$ for $z_{\beta}=\beta h \in \mathcal{G}_{h}$ and $\beta \in \mathbb{Z}^{N}$, and $p_{0}^{1}\left(x_{1}, \ldots, x_{i}, \ldots, x_{N}\right)=$ $p_{0}^{1}\left(x_{1}, \ldots,-x_{i}, \ldots, x_{N}\right)$ for $x_{i} \in \mathbb{R}$ and $i=1, \ldots, N$.

4.2. Discretizations of the local operator $L^{\sigma}$. The operator $L^{\sigma}$ given by (1.4) is a local, self-adjoint, and possibly degenerate operator that can be written as

$$
L^{\sigma}[\psi](x):=\operatorname{tr}\left(\sigma \sigma^{T} D^{2} \psi(x)\right)=\sum_{i=1}^{P} \sigma_{i}^{T} D^{2} \psi(x) \sigma_{i}=\sum_{i=1}^{P}\left(\sigma_{i}^{T} D\right)^{2} \psi(x)
$$


where $\sigma=\left(\sigma_{1}, \ldots, \sigma_{P}\right) \in \mathbb{R}^{N \times P}$ and $\sigma_{i} \in \mathbb{R}^{N}$. For $\eta>0$, we approximate $L^{\sigma}$ by

$$
L_{\eta}^{\sigma}[\psi](x):=\sum_{i=1}^{P} \frac{\psi\left(x+\sigma_{i} \eta\right)+\psi\left(x-\sigma_{i} \eta\right)-2 \psi(x)}{\eta^{2}} .
$$

In general $x+\sigma_{i} \eta \notin \mathcal{G}_{h}$, not even when $x \in \mathcal{G}_{h}$, and hence this discretization is in the form (FD) only for special choices of $\sigma$. We can overcome this problem by replacing $\psi$ by its interpolant on $\mathcal{G}_{h}$,

$$
L_{\eta, h}^{\sigma}[\psi](x)=\sum_{i=1}^{P} \frac{I_{h}^{1}[\psi]\left(x+\sigma_{i} \eta\right)+I_{h}^{1}[\psi]\left(x-\sigma_{i} \eta\right)-2 \psi(x)}{\eta^{2}}
$$

where $I_{h}^{1}$ denotes the first order Lagrange interpolation defined in (4.1) for $k=1$. This type of discretizations have been studied before e.g. in [16, 35].

Remark 4.1. (a) If $\eta=h$ and $\sigma_{i}=e_{i}$, the standard basis in $\mathbb{R}^{N}$, then

$$
L^{\sigma}=\Delta \quad \text { and } \quad L_{h}^{\sigma}=L_{h, h}^{\sigma}=\Delta_{h},
$$

where $\Delta_{h}$ is the classical second order finite difference approximation

$$
\Delta_{h} \psi(x):=\sum_{i=1}^{N} \frac{\psi\left(x+h e_{i}\right)-2 \psi(x)+\psi\left(x-h e_{i}\right)}{h^{2}} .
$$

(b) By a coordinate transformation $x=A y$ (diagonalization), $L^{\sigma}$ can always be transformed into

$$
L^{I_{0}} \quad \text { where } \quad I_{0}:=\left[\begin{array}{l|l}
I & 0 \\
\hline 0 & 0
\end{array}\right] \in \mathbb{R}^{N \times N},
$$

where $I$ is an identity matrix. $L^{I_{0}}$ corresponds to a Laplacian operator in $\mathbb{R}^{M}$ for some $M \leq N$. In the transformed coordinates $y, L^{I_{0}}=\Delta_{\mathbb{R}^{M}}$, the $\mathbb{R}^{M}$-Laplacian, for some $M \leq N$, and again $L_{h}^{I_{0}}=L_{h, h}^{I_{0}}=\Delta_{\mathbb{R}^{M}, h}$ (see [35]).

We have the following general result.

Lemma 4.2. Let $h, \eta>0, h=o(\eta)$, and $L^{\sigma}$ be defined by (1.4). The family of operators $\left\{L_{\eta, h}^{\sigma}\right\}_{\eta, h>0}$ given by (4.5) is an admissible approximation of $L^{\sigma}$ with Local Truncation Error $O\left(\frac{h^{2}}{\eta^{2}}+\eta^{2}\right)$ (or $O(h)$ with the optimal $\left.\eta=\sqrt{h}\right)$.

Lemma 4.2 is close to results e.g. in [32], but below we give a proof for completeness. For the discretization $\Delta_{h}$ we have the following result.

Lemma 4.3. Let $h>0$. The family of operators $\left\{\Delta_{h}\right\}_{h>0}$ given by (4.6) is an admissible approximation of $\Delta$ with Local Truncation Error $O\left(h^{2}\right)$.

Admissibility and the improved (and classical!) rate follows as in the proof of Lemma 4.2 since there is no interpolation now. 
Proof of Lemma 4.2. We show that $L_{\eta, h}^{\sigma}$ can be written in the finite difference form (FD). For simplicity, let us set $\sigma_{-i}=-\sigma_{i}$ and let $x_{\alpha} \in \mathcal{G}_{h}$. Then

$$
\begin{aligned}
L_{\eta, h}^{\sigma}[\psi]\left(x_{\alpha}\right) & =\sum_{i=1}^{P} \frac{I_{h}^{1}[\psi]\left(x_{\alpha}+\sigma_{i} \eta\right)+I_{h}^{1}[\psi]\left(x_{\alpha}-\sigma_{i} \eta\right)-2 \psi\left(x_{\alpha}\right)}{\eta^{2}} \\
& =\sum_{0<|i| \leq P}\left(\sum_{\beta} \psi\left(z_{\beta}\right) p_{0}^{1}\left(x_{\alpha}+\sigma_{i} \eta-z_{\beta}\right)-\psi\left(x_{\alpha}\right)\right) \frac{1}{\eta^{2}} \\
& =\sum_{0<|i| \leq P}\left(\sum_{\gamma} \psi\left(x_{\alpha}+z_{\gamma}\right) p_{0}^{1}\left(\sigma_{i} \eta-z_{\gamma}\right)-\psi\left(x_{\alpha}\right)\right) \frac{1}{\eta^{2}} \\
& =\sum_{\gamma \neq 0}\left(\psi\left(x_{\alpha}+z_{\gamma}\right)-\psi\left(x_{\alpha}\right)\right)\left(\frac{1}{\eta^{2}} \sum_{0<|i| \leq P} p_{0}^{1}\left(\sigma_{i} \eta-z_{\gamma}\right)\right) .
\end{aligned}
$$

The weights are $\omega_{\eta, h, \beta}=\frac{1}{\eta^{2}} \sum_{0<|i| \leq P} p_{0}^{1}\left(\sigma_{i} \eta-z_{\beta}\right)$, and we immediately find that $0 \leq \omega_{\eta, h, \beta}=\omega_{\eta, h,-\beta}$ since $0 \leq p_{0}^{1}$ is even, $\sigma_{-i}=-\sigma_{i}$, and $z_{-\beta}=-z_{\beta}$. Moreover,

$$
\sum_{\beta \neq 0} \omega_{\eta, h, \beta}=\frac{1}{\eta^{2}} \sum_{0<|i| \leq P} \sum_{\beta \neq 0} p_{\beta}^{1}\left(\sigma_{i} \eta\right)=\frac{1}{\eta^{2}} \sum_{0<|i| \leq P}\left(1-p_{0}^{1}\left(\sigma_{i} \eta\right)\right) \leq \frac{2 P}{\eta^{2}}<+\infty .
$$

To show consistency we split the error in the following way,

$$
\left\|L^{\sigma} \psi-L_{\eta, h}^{\sigma}[\psi]\right\|_{L^{1}\left(\mathbb{R}^{N}\right)} \leq\left\|L^{\sigma} \psi-L_{\eta}^{\sigma}[\psi]\right\|_{L^{1}\left(\mathbb{R}^{N}\right)}+\left\|L_{\eta}^{\sigma}[\psi]-L_{\eta, h}^{\sigma}[\psi]\right\|_{L^{1}\left(\mathbb{R}^{N}\right)}
$$

where $L_{\eta}^{\sigma}[\psi]$ is given by (4.4). The first term on the right hand side is the classical error of a second order approximation of second derivatives,

$$
\left\|L^{\sigma} \psi-L_{\eta}^{\sigma}[\psi]\right\|_{L^{1}\left(\mathbb{R}^{N}\right)} \leq C\left\|D^{4} \psi\right\|_{L^{1}\left(\mathbb{R}^{N}\right)} \eta^{2}|\sigma|^{4} .
$$

For the second term, we have (cf. (4.2))

$$
\begin{aligned}
\left\|L_{\eta}^{\sigma}[\psi]-L_{\eta, h}^{\sigma}[\psi]\right\|_{L^{1}\left(\mathbb{R}^{N}\right)} & =\frac{1}{\eta^{2}}\left\|\sum_{0<|i| \leq P}\left(I_{h}^{1}[\psi]\left(\cdot+\sigma_{i} \eta\right)-\psi\left(\cdot+\sigma_{i} \eta\right)\right)\right\|_{L^{1}\left(\mathbb{R}^{N}\right)} \\
& \leq \frac{1}{\eta^{2}} \sum_{0<|i| \leq P}\left\|I_{h}^{1}[\psi]\left(\cdot+\sigma_{i} \eta\right)-\psi\left(\cdot+\sigma_{i} \eta\right)\right\|_{L^{1}\left(\mathbb{R}^{N}\right)} \\
& =\frac{1}{\eta^{2}} \sum_{0<|i| \leq P} C\left\|D^{2} \psi\right\|_{L^{1}\left(\mathbb{R}^{N}\right)} h^{2}=O\left(\frac{h^{2}}{\eta^{2}}\right) .
\end{aligned}
$$

Thus, for any choice of $h=o(\eta)$ we get a consistent scheme. Moreover, one can observe that the last two estimates also hold in $L^{\infty}$ by a trivial adaptation. By Proposition 3.2 it then follows that the uniform integrability condition (UL) holds. In view of Lemma 3.1, the scheme is admissible (cf. Definition 3.1) and the proof is complete.

4.3. Discretizations of the singular nonlocal operator $\mathcal{L}_{r}^{\mu}$. We present discretizations of the singular/unbounded part of the nonlocal operator (recall Remark 1.1)

$$
\mathcal{L}_{r}^{\mu}[\psi](x)=\int_{0<|z| \leq r}(\psi(x+z)-\psi(x)-z \cdot D \psi(x)) \mathrm{d} \mu(z), \quad r \in[0,1] .
$$

We start with the trivial discretization where we discretize $\mathcal{L}_{r}^{\mu}$ by

$$
\mathcal{L}^{h}[\psi](x) \equiv 0
$$


This crude discretization is computationally efficient, and depending on the order of the operator and the other discretizations involved, the error could be satisfactory.

Lemma 4.4. Assume $\left(\mathrm{A}_{\mu}\right), h \leq r=o_{h}(1)$, and $\mathcal{L}_{r}^{\mu}$ is given by (4.8). Then $\left\{\mathcal{L}^{h}\right\}_{h>0}$ given by (4.9) is an admissible approximation of $\mathcal{L}_{r}^{\mu}$. Moreover, if also $\left(\mathrm{A}_{\mu_{\alpha}}\right)$ holds, then the Local Truncation Error is $O\left(r^{2-\alpha}\right)$.

Proof. Since $\mathcal{L}^{h}=\mathcal{L}^{\nu_{h}}$ with $\nu_{h} \equiv 0$, it is a discretization of the form (FD) in the class $\left(\mathrm{A}_{\nu}\right)$. It is consistent by the dominated convergence theorem,

$$
\begin{aligned}
\left\|\mathcal{L}_{r}^{\mu}[\psi]-\mathcal{L}^{h}[\psi]\right\|_{L^{1}\left(\mathbb{R}^{N}\right)} & =\left\|\mathcal{L}_{r}^{\mu}[\psi]\right\|_{L^{1}\left(\mathbb{R}^{N}\right)} \\
& \leq \frac{1}{2}\left\|D^{2} \psi\right\|_{L^{1}\left(\mathbb{R}^{N}\right)} \int_{0<|z| \leq r}|z|^{2} \mathrm{~d} \mu(z) \rightarrow 0 \quad \text { as } \quad h \rightarrow 0^{+} .
\end{aligned}
$$

If also $\left(\mathrm{A}_{\mu_{\alpha}}\right)$ holds, then the LTE is $O\left(r^{2-\alpha}\right)$. Moreover (UL) also holds since $\sup _{h<1} \sum_{\beta \neq 0}\left(\left|z_{\beta}\right|^{2} \wedge 1\right) \omega_{\beta, h}=0<+\infty$.

We now show how to get a more accurate discretization of $\mathcal{L}_{r}^{\mu}$ through the adapted vanishing viscosity discretization [5, 26, 53]: Approximate $\mathcal{L}_{r}^{\mu}$ by a local second order operator and then discretize. To do this, note that by Taylor's theorem,

$$
\psi(x+z)-\psi(x)-z \cdot D \psi(x)=\sum_{2 \leq|\beta| \leq 3} \frac{1}{\beta !} D^{\beta} \psi(x) z^{\beta}+\sum_{|\beta|=4} R_{\beta}(x, z) z^{\beta} .
$$

where $R_{\beta}(x, z)=\frac{|\beta|}{\beta !} \int_{0}^{1}(1-s)^{|\beta-1|} D^{\beta} \psi(x+s z) \mathrm{d} s$. Since terms with $|\beta|=3$ are odd and the measure $\mu$ is symmetric,

$$
\mathcal{L}_{r}^{\mu}[\psi](x)=T_{2}^{r}(x)+0+R_{4}^{r}(x),
$$

where $R_{4}^{r}(x)=\sum_{|\beta|=4} \int_{|z|<r} R_{\beta}(x, z) z^{\beta} \mathrm{d} \mu(z)$,

$$
T_{2}^{r}(x)=\sum_{|\beta|=2} \frac{D^{\beta} \psi(x)}{\beta !} \int_{|z|<r} z^{\beta} \mathrm{d} \mu(z)=\frac{1}{2} \operatorname{tr}\left[Z D^{2} \psi(x)\right],
$$

and $Z_{i j}=\int_{|z|<r} z_{i} z_{j} \mathrm{~d} \mu(z)$. Observe that $Z=Z(r)$ is a symmetric and positive semidefinite matrix, $\xi^{T} Z \xi=\int_{|z|<r} \xi^{T}\left(z z^{T}\right) \xi \mathrm{d} \mu(z)=\int_{|z|<r}(\xi \cdot z)^{2} \mathrm{~d} \mu(z) \geq 0$ for $\xi \in \mathbb{R}^{N}$, and that $Z(r) \rightarrow 0$ as $r \rightarrow 0$ by the dominated convergence theorem. Hence it has a square root $\sqrt{Z}$ with entries $(\sqrt{Z})_{i j}$ and columns $(\sqrt{Z})_{i}$ (our notation). This allows us to write $T_{2}^{r}$ as a sum of directional derivatives

$$
T_{2}^{r}(x)=\frac{1}{2} \sum_{i=1}^{N}\left((\sqrt{Z})_{i} \cdot D\right)^{2} \psi(x) .
$$

This is an operator of the form $L^{\sigma}$ with $\sigma_{i}=(\sqrt{Z})_{i}, i=1, \ldots, N$ (cf. (4.3)), and we discretize it as in Section 4.2:

$$
\mathcal{L}^{\eta, h}[\psi](x)=\sum_{i=1}^{P} \frac{I_{h}^{1}[\psi]\left(x+(\sqrt{Z})_{i} \eta\right)+I_{h}^{1}[\psi]\left(x-(\sqrt{Z})_{i} \eta\right)-2 \psi(x)}{\eta^{2}}
$$

Note that (4.10) depends on $r$ through the terms $(\sqrt{Z})_{i}$, and that (linear) interpolation is needed since $x+(\sqrt{Z})_{i} \eta$ do not belong to the grid $\mathcal{G}_{h}$ in general.

Lemma 4.5. Assume $\left(\mathrm{A}_{\mu}\right), h, \eta, r>0, h=o(\eta), h \leq r=o_{h}(1)$, and $\mathcal{L}_{r}^{\mu}$ is defined by (4.8). Then the family $\left\{\mathcal{L}^{\eta, h}\right\}_{h, \eta>0}$ given by (4.10) is an admissible approximation of $\mathcal{L}_{r}^{\mu}$ with Local Truncation Error $O\left(\frac{h^{2}}{\eta^{2}}+\eta^{2}|Z(r)|^{2}+r^{2}\right)$. Moreover, if also $\left(\mathrm{A}_{\mu_{\alpha}}\right)$ holds, then the LTE is $O\left(\frac{h^{2}}{\eta^{2}}+\eta^{2} r^{4-2 \alpha}+r^{4-\alpha}\right)$. 
In general, the optimal choice will turn out to be $\eta=r=h^{\frac{1}{2}}$ which gives a linear rate $O(h)$. If also $\left(\mathrm{A}_{\mu_{\alpha}}\right)$ holds, the optimal choice will turn out to be $r=h^{\frac{1}{2}}$ and $\eta=h^{\frac{\alpha}{4}}$ which gives the superlinear rate $O\left(h^{2-\frac{\alpha}{2}}\right)$. The proof will be given below. Now we show how this rate can be improved when the measure $\mu$ satisfies stronger symmetry assumptions.

Definition 4.1. A Borel measure $\mu$ is symmetric in coordinate directions if $\mu(A)=\mu\left(T_{x_{i}}(A)\right)$ for all $i=1, \ldots, N$ and Borel sets $A$, where $T_{x_{i}}(A)=$ $\left\{\left(x_{1}, \ldots,-x_{i}, \ldots, x_{N}\right):\left(x_{1}, \ldots, x_{i}, \ldots, x_{N}\right) \in A\right\}$.

Remark 4.6. Obviously radial symmetry $\Rightarrow$ symmetry in coordinate directions $\Rightarrow$ symmetry $(\mu(A)=\mu(-A))$. All reverse implications are false. If e.g. $\mathrm{d} \mu\left(z_{1}, z_{2}\right)=$ $\operatorname{sign}\left(z_{1} z_{2}\right)^{+} \mathrm{d} z_{1} \mathrm{~d} z_{2}$, then $\mu$ is symmetric but not symmetric in coordinate directions.

If $\mu$ is symmetric in coordinate directions, then mixed derivatives vanish and $T_{2}^{r}=\frac{1}{2} \sum_{i=1}^{N} \frac{\partial^{2} \psi}{\partial x_{i}^{2}} \int_{|z|<r} z_{i}^{2} \mathrm{~d} \mu(z)$. A natural difference approximation of $\mathcal{L}_{r}^{\mu}$ is then

$$
\mathcal{L}^{h}[\psi](x):=\frac{1}{2} \sum_{i=1}^{N} \frac{\psi\left(x+e_{i} h\right)+\psi\left(x-e_{i} h\right)-2 \psi(x)}{h^{2}} \int_{|z|<r} z_{i}^{2} \mathrm{~d} \mu(z) .
$$

If $\mu$ is also radially symmetric we even have $\int_{|z|<r} z_{i}^{2} \mathrm{~d} \mu(z)=\frac{1}{N} \int_{|z|<r}|z|^{2} \mathrm{~d} \mu(z)$.

Lemma 4.7. Assume $\left(\mathrm{A}_{\mu}\right), \mu$ is symmetric in coordinate directions, $h, r>0$, $h \leq r=o_{h}(1)$, and $\mathcal{L}_{r}^{\mu}$ is defined by (4.8). Then the family $\left\{\mathcal{L}^{h}\right\}_{h>0}$ given by $(4.11)$ is an admissible approximation of $\mathcal{L}_{r}^{\mu}$ with Local Truncation Error $O\left(h^{2}+r^{2}\right)$. Moreover, if also $\left(\mathrm{A}_{\mu_{\alpha}}\right)$ holds, then the LTE is $O\left(h^{2} r^{2-\alpha}+r^{4-\alpha}\right)$.

The optimal choice will turn out to be $r=h$ which gives a quadratic rate $O\left(h^{2}\right)$ or superquadratic rate $O\left(h^{4-\alpha}\right)$ if also $\left(\mathrm{A}_{\mu_{\alpha}}\right)$ holds. The proof is similar to the proof of Lemma 4.5.

Proof of Lemma 4.5. Since $\mathcal{L}^{\eta, h}$ is in the form of (4.5), it is already in finite difference form (FD). By Lemma 4.2 it is in the class $\left(\mathrm{A}_{\nu}\right)$. We now check consistency.

$$
\left\|\mathcal{L}_{r}^{\mu}[\psi]-\mathcal{L}^{\eta, h}[\psi]\right\|_{L^{1}\left(\mathbb{R}^{N}\right)} \leq\left\|T_{2}^{r}-\mathcal{L}^{\eta, h}[\psi]\right\|_{L^{1}\left(\mathbb{R}^{N}\right)}+\left\|R_{4}^{r}\right\|_{L^{1}\left(\mathbb{R}^{N}\right)},
$$

and by Lemma 4.2 and inequality (4.7) with $\sigma_{i}=(\sqrt{Z})_{i}$, the definition of $R_{4}^{r}$, and Fubini,

$$
\begin{aligned}
\left\|T_{2}^{r}-\mathcal{L}^{\eta, h}[\psi]\right\|_{L^{1}\left(\mathbb{R}^{N}\right)} & \leq C\left\|D^{2} \psi\right\|_{L^{1}\left(\mathbb{R}^{N}\right)} \frac{h^{2}}{\eta^{2}}+C\left\|D^{4} \psi\right\|_{L^{1}\left(\mathbb{R}^{N}\right)} \eta^{2}|Z|^{2} \\
& =O\left(\frac{h^{2}}{\eta^{2}}+\eta^{2}|Z|^{2}\right) \\
\left\|R_{4}^{r}\right\|_{L^{1}\left(\mathbb{R}^{N}\right)} & \leq C\left\|D^{4} \psi\right\|_{L^{1}\left(\mathbb{R}^{N}\right)} \int_{|z|<r}|z|^{4} \mathrm{~d} \mu(z)=O\left(r^{2}\right),
\end{aligned}
$$

leading to the desired Local Truncation Error. Since $\left|Z_{i}\right| \leq C \int_{|z|<r}|z|^{2} \mathrm{~d} \mu(z)$, the modifications when also $\left(\mathrm{A}_{\mu_{\alpha}}\right)$ holds are obvious. Once again, the estimates also hold in $L^{\infty}$ with a trivial adaptation and thus Proposition 3.2 ensures that (UL) is satisfied.

4.4. Discretizations of the bounded nonlocal operator $\mathcal{L}^{\mu, r}$. We present discretization of the bounded/nonsingular part of the nonlocal operator, i.e. of

$$
\mathcal{L}^{\mu, r}[\psi](x):=\int_{|z|>r}(\psi(x+z)-\psi(x)) \mathrm{d} \mu(z), \quad r \in[0,1] .
$$

Note that $\mathcal{L}^{\mu, r}$ is an operator in the form $\left(\mathrm{A}_{\mu}\right)$ with measure $\mathrm{d} \mu_{r}(z)=\mathbf{1}_{|z|>r} \mathrm{~d} \mu(z)$. 
4.4.1. Midpoint quadrature rule. The idea is to approximate $\psi$ on each cube $z_{\beta}+R_{h}$ by its midpoint value $\psi\left(z_{\beta}\right)$. This gives the following quadrature formula for the cube,

$$
\int_{z_{\beta}+R_{h}}(\psi(x+z)-\psi(x)) \mathrm{d} \mu(z) \simeq\left(\psi\left(x+z_{\beta}\right)-\psi(x)\right) \mu\left(z_{\beta}+R_{h}\right),
$$

and a discretization of $\mathcal{L}^{\mu, r}$ given by

$$
\mathcal{L}^{h}[\psi](x)=\sum_{\beta \neq 0}\left(\psi\left(x+z_{\beta}\right)-\psi(x)\right) \mu\left(z_{\beta}+R_{h}\right) .
$$

Lemma 4.8. Assume $\left(\mathrm{A}_{\mu}\right), h \leq r=o_{h}(1)$, and $\mathcal{L}^{\mu, r}$ is defined by (4.12). The family $\left\{\mathcal{L}^{h}\right\}_{h>0}$ given by (4.13) is an admissible approximation of $\mathcal{L}^{\mu, r}$. Moreover, if also $\left(\mathrm{A}_{\mu_{\alpha}}\right)$ holds, then the Local Truncation Error is $O\left(h+r^{2-\alpha}\right)$.

The result was proved in Lemma 5.3 and 5.4 in [35]. Note that since $\mu$ is not translation invariant as the Lebesgue measure, the midpoint rule is no longer a second order method in general. Moreover, the LTE is dominated by the contribution from the integral over $|z|>1$ and can therefore not be improved by assuming $\left(\mathrm{A}_{\mu_{\alpha}}\right)$.

Remark 4.9. Lemma 4.8 is consistent with the numerical experiments in Section 5 for $\alpha>1$, while for $\alpha<1$ the numerical results are better. Note that if $N=1, \alpha=1$ and $r=h$, then the midpoint rule coincides with the second order discretization of Section 4.5 below. This time the observed LTE is $O\left(h^{2}\right)$.

4.4.2. Quadrature from interpolation - general $\mu$. The idea is to replace $\psi(x+z)-$ $\psi(x)$ by a Lagrange polynomial interpolant on $\mathcal{G}_{h}$ (defined in Section 4.1),

$$
I_{h}^{k}[\psi(x+\cdot)-\psi(x)](z)=\sum_{\beta \neq 0}\left(\psi\left(x+z_{\beta}\right)-\psi(x)\right) p_{\beta}^{k}(z),
$$

and integrate with respect to the measure $\mu$ to obtain the discretization

$$
\begin{aligned}
\mathcal{L}^{h}[\psi](x) & =\int_{|z|>r} I_{h}^{k}[\psi(x+\cdot)-\psi(x)](z) \mathrm{d} \mu(z) \\
& =\sum_{\beta \neq 0}\left(\psi\left(x+z_{\beta}\right)-\psi(x)\right) \int_{|z|>r} p_{\beta}^{k}(z) \mathrm{d} \mu(z) .
\end{aligned}
$$

This is a classical idea that has been used for nonlocal operators before, e.g. [8, 50].

To have a more understandable presentation, we divide the proof of admissibility into several lemmas. We begin by noting that the operator (4.14) obviously is in the finite difference form (FD) with symmetric weights

$$
\omega_{\beta, h}=\int_{|z|>r} p_{\beta}^{k}(z) \mathrm{d} \mu(z), \quad \beta \in \mathbb{Z}^{N} .
$$

However, the nonnegativity of the weights is only guaranteed when $k=0$ and $k=1$ since these are the only cases where the basis functions $p_{\beta}^{k}$ are all nonnegative.

Lemma 4.10. Assume $\left(\mathrm{A}_{\mu}\right), h, r>0$, and $k=0$ or $k=1$. Then the family $\left\{\mathcal{L}^{h}\right\}_{h>0}$ given by (4.14) is in the class $\left(\mathrm{A}_{\nu}\right)$.

Proof. In view of the above discussion and Lemma 3.1, it remains to check that

$$
\sum_{\beta} \omega_{\beta, h}=\sum_{\beta} \int_{|z|>r} p_{\beta}^{k}(z) \mathrm{d} \mu(z)=\int_{|z|>r} \sum_{\beta} p_{\beta}^{k}(z) \mathrm{d} \mu(z)=\int_{|z|>r} \mathrm{~d} \mu(z)<+\infty .
$$

The proof is complete. 
Remark 4.11. Note that $p_{\beta}^{k} \geq 0$ implies $\omega_{\beta, h} \geq 0$, but the other implication depends on $\mu$ and is not true in general. But if $\mu$ were the Lebesgue measure supported on a cube, then our quadrature would coincide with the Newton-Cotes quadratures which are known to have nonnegative weights for orders $k \leq 6$.

Moreover, if $\mathrm{d} \mu(z)=\frac{\mathrm{d} z}{|z|^{N+\alpha}}$ (fractional Laplace), then explicit nonnegative weights are found and presented in a nice way in [50] for $N=1, k=1$ and $k=2$. In this case quadratic interpolation combined with (4.11) yields an admissible discretization of $-(-\Delta)^{\frac{\alpha}{2}}$ with a LTE of $O\left(h^{3-\alpha}\right)$. Numerical evidence for this rate is given in Section 5 .

The following result on local truncation error is valid for any $k \in \mathbb{N}$.

Lemma 4.12. Assume $\left(\mathrm{A}_{\mu}\right), h \leq r=o_{h}(1), k \geq 0$, and $\mathcal{L}^{\mu, r}$ is defined by (4.12). Then the family $\left\{\mathcal{L}^{h}\right\}_{h>0}$ given by (4.14) has Local Truncation Error

$$
\left\|\mathcal{L}^{\mu, r}[\psi]-\mathcal{L}^{h}[\psi]\right\|_{L^{p}} \leq C h^{k+1}\left\|D^{k+1} \psi\right\|_{L^{p}} \mu(\{|z|>r\}), \quad p \in\{1, \infty\} .
$$

Moreover, if also $\left(\mathrm{A}_{\mu_{\alpha}}\right)$ holds, then the LTE is $O\left(h^{k+1} r^{-\alpha}\right)$.

Proof. We use the notation $P_{\psi}(x, z)=I_{h}^{k}[\psi(x+\cdot)-\psi(x)](z)$ and use (4.2) to get

$$
\begin{aligned}
\left\|\mathcal{L}^{\mu, r}[\psi]-\mathcal{L}^{h}[\psi]\right\|_{L^{p}\left(\mathbb{R}^{N}\right)} & \leq \int_{|z|>r}\left\|P_{\psi}(\cdot, z)-(\psi(\cdot+z)-\psi(\cdot))\right\|_{L^{p}\left(\mathbb{R}^{N}\right)} \mathrm{d} \mu(z) \\
& \leq C\left\|D^{k+1} \psi\right\|_{L^{p}\left(\mathbb{R}^{N}\right)} h^{k+1} \int_{|z|>r} \mathrm{~d} \mu(z) .
\end{aligned}
$$

The proof is complete.

To be consistent we need to impose that $h^{k+1} \mu(\{|z|>r\}) \rightarrow 0$ as $h \rightarrow 0$. When also $\left(\mathrm{A}_{\mu_{\alpha}}\right)$ holds, this is always satisfied for $k \geq 1$ and $r=o_{h}(1)$, while for $k=0$ we need $h r^{-\alpha}=o_{h}(1)$. We are now in a position to state and prove admissibility for the cases $k=0$ and $k=1$.

Lemma 4.13. Assume $\left(\mathrm{A}_{\mu}\right), h \leq r=o_{h}(1)$, and $\mathcal{L}^{\mu, r}$ is defined by (4.12). If either $k=0$ and $h=o_{r}\left(\frac{1}{\mu(\{|z|>r\})}\right)$ or $k=1$, then the family $\left\{\mathcal{L}^{h}\right\}_{h>0}$ given by (4.13) is an admissible approximation of $\mathcal{L}^{\mu, r}$.

Proof. By the above discussion and Lemma 4.10, $\left\{\mathcal{L}^{h}\right\}_{h>0}$ is a (FD) type discretization in the class $\left(\mathrm{A}_{\nu}\right)$. For consistency we use the error estimate (4.15). When $k=0$ we can conclude from the extra assumption $h=o_{r}\left(\frac{1}{\mu(\{|z|>r\})}\right)$. When $k=1$, we observe that

$$
h^{k+1} \int_{|z|>r} \mathrm{~d} \mu(z)=\int_{|z|>r} h^{2} \mathrm{~d} \mu(z) \leq \int_{|z|>0}|z|^{2} \wedge r^{2} \mathrm{~d} \mu(z)
$$

and conclude by the Dominated Convergence Theorem since $|z|^{2} \wedge r^{2} \rightarrow 0$ pointwise as $r \rightarrow 0^{+}$and $|z|^{2} \wedge r^{2} \leq|z|^{2} \wedge 1(r \leq 1)$ which is integrable by $\left(\mathrm{A}_{\mu}\right)$. Uniform integrability (UL) follows from Proposition 3.2 and the $L^{\infty}$ version of the above estimate.

For admissibility and higher order interpolation, see Remark 4.11.

4.4.3. Quadrature from interpolation - absolute continuous $\mu$. If $\mu$ is absolutely continuous with respect to the Lebesgue measure $\mathrm{d} z$ with density also called $\mu(z)$, then (cf. [53]) we can approximate $\mathcal{L}^{\mu, r}$ by

$$
\begin{aligned}
\mathcal{L}^{h}[\psi](x) & :=\int_{|z|>r} I_{h}^{k}[(\psi(x+\cdot)-\psi(x)) \mu(\cdot)](z) \mathrm{d} z \\
& =\sum_{\left|z_{\beta}\right|>r}\left(\psi\left(x+z_{\beta}\right)-\psi(x)\right) \mu\left(z_{\beta}\right) \int_{|z|>r} p_{\beta}^{k}(z) \mathrm{d} z .
\end{aligned}
$$


Note that (4.16) is in the finite difference form (FD) with symmetric weights

$$
\omega_{\beta, h}=\mu\left(z_{\beta}\right) \int_{|z|>r} p_{\beta}^{k}(z) \mathrm{d} z \quad \text { for } \quad\left|z_{\beta}\right|>r \quad \text { and } \quad \omega_{\beta, h}=0 \quad \text { otherwise. }
$$

The weights are nonnegative for $k \leq 6$ since $\mu \geq 0$ and $\tilde{\omega}_{\beta, h}=\int_{\mathbb{R}^{N}} p_{\beta}^{k}(z) \mathrm{d} z$ coincides with Newton-Cotes quadrature weights that are known to be nonnegative. As we will describe later, this discretization can also be combined with (4.11) to further improve the order of accuracy.

Lemma 4.14. Assume $\left(\mathrm{A}_{\mu}\right), 0 \leq k \leq 6, h, r>0$, and $\mathrm{d} \mu(z)=\mu(z) \mathrm{d} z$ for $a$ density $\mu \in C_{b}\left(\mathbb{R}^{N} \backslash B_{r}\right)$. Then the family $\left\{\mathcal{L}^{h}\right\}_{h>0}$ given by (4.16) is in the class $\left(\mathrm{A}_{\nu}\right)$.

Proof. By the previous discussion we know that $\mathcal{L}^{h}$ is of the form (FD) with symmetric nonnegative weights, so by Lemma 3.1, we just need to check:

$$
\begin{aligned}
\sum_{\left|z_{\beta}\right|>r} \mu\left(z_{\beta}\right) \int_{|z|>r} p_{\beta}^{k}(z) \mathrm{d} \mu(z) & \leq\|\mu\|_{L^{\infty}\left(\mathbb{R}^{N} \backslash B_{r}\right)} \int_{|z|>r} \sum_{\beta} p_{\beta}^{k}(z) \mathrm{d} \mu(z) \\
& =\|\mu\|_{L^{\infty}\left(\mathbb{R}^{N} \backslash B_{r}\right)} \int_{|z|>r} \mathrm{~d} \mu(z)<+\infty
\end{aligned}
$$

The proof is complete.

Since also $\mu$ is interpolated now, the local truncation error will depend on the regularity of $\mu$. We state the result using standard Sobolov spaces $W^{k, 1}$ and $W^{k, \infty}$.

Lemma 4.15. Assume $\left(\mathrm{A}_{\mu}\right), 0 \leq k \leq 6, h, r>0$, and that $\mathrm{d} \mu(z)=\mu(z) \mathrm{d} z$ for $a$ density $\mu \in C\left(\mathbb{R}^{N} \backslash B_{r}\right) \cap W^{k+1,1}\left(\mathbb{R}^{N} \backslash B_{r}\right)$. If $\mathcal{L}^{\mu, r}$ is defined by (4.12) and $\left\{\mathcal{L}^{h}\right\}_{h>0}$ by $(4.16)$, then there is a constant $C>0$ such that for $p \in\{1, \infty\}$,

$$
\left\|\mathcal{L}^{\mu, r}[\psi]-\mathcal{L}^{h}[\psi]\right\|_{L^{p}\left(\mathbb{R}^{N}\right)} \leq C h^{k+1}\|\psi\|_{W^{k+1, p}\left(\mathbb{R}^{N}\right)}\|\mu\|_{W^{k+1,1}\left(\mathbb{R}^{N} \backslash B_{r}\right)} .
$$

Proof. Let $Q_{\alpha}:=z_{\alpha}+\frac{k h}{2}(-1,1]^{N}$ for $\alpha \in \mathbb{Z}^{N}, y_{i}^{\alpha}$ denote the $(k+1)^{N}$ gridpoints in $Q_{\alpha} \cap \mathcal{G}_{h}$, and $F(x, z)=(\psi(x+z)-\psi(x)) \mu(z)$. Note that then $I_{h}^{k} \psi\left(y_{i}^{\alpha}\right)=\psi\left(y_{i}^{\alpha}\right)$,

$$
\bigcup_{\alpha \in k \mathbb{Z}^{N}} Q_{\alpha}=\mathbb{R}^{N}, \quad \text { and } \quad \mathcal{L}^{h}[\psi](x):=\int_{|z|>r} I_{h}^{k}[F(x, \cdot)](z) \mathrm{d} z .
$$

Using Taylor expansions with integral remainder terms at every point $y_{i}^{\alpha}$, we get

$$
\begin{aligned}
& \left|\mathcal{L}^{h}[\psi](x)-\mathcal{L}^{\mu, r}[\psi](x)\right| \leq \sum_{\alpha \in k \mathbb{Z}^{N},\left|z_{\alpha}\right|>r} \int_{Q_{\alpha}}\left|I_{h}^{k}[F(x, \cdot)](z)-F(x, z)\right| \mathrm{d} z \\
& \leq h^{k+1} \sum_{i=1}^{(k+1)^{N}} \sum_{\alpha \in k \mathbb{Z}^{N},\left|z_{\alpha}\right|>r} \int_{Q_{\alpha}} \int_{0}^{1}\left|D_{z}^{k+1} F\left(x, z(1-s)+s y_{i}^{\alpha}\right)\right| \mathrm{d} s \mathrm{~d} z .
\end{aligned}
$$

By Fubini, the definition of $F$, and the chain rule, for $p=\{1, \infty\}$ it follows that

$$
\begin{aligned}
& \left\|\mathcal{L}^{h}[\psi]-\mathcal{L}^{\mu, r}[\psi]\right\|_{L^{p}\left(\mathbb{R}^{N}\right)} \\
& \leq h^{k+1} \sum_{i=1}^{(k+1)^{N}} \sum_{\alpha \in k \mathbb{Z}^{N},\left|z_{\alpha}\right|>r} \int_{Q_{\alpha}} \int_{0}^{1}\left\|D_{z}^{k+1} F\left(\cdot, z(1-s)+s y_{i}^{\alpha}\right)\right\|_{L^{p}\left(\mathbb{R}^{N}\right)} \mathrm{d} s \mathrm{~d} z \\
& \leq C h^{k+1}\|\psi\|_{W^{k+1, p}\left(\mathbb{R}^{N}\right)} \sum_{i=1}^{(k+1)^{N}} \sum_{\alpha \in k \mathbb{Z}^{N},\left|z_{\alpha}\right|>r} \sum_{l=0}^{k+1} \int_{0}^{1} \int_{Q_{\alpha}}\left|D^{l} \mu\left(z(1-s)+s y_{i}^{\alpha}\right)\right| \mathrm{d} z \mathrm{~d} s
\end{aligned}
$$


Now we do the change of variables $y=z(1-s)+s y_{i}^{\alpha}$, which has the change in measure $\mathrm{d} y=(1-s)^{N} \mathrm{~d} z$ and maps $Q_{\alpha}$ into $\tilde{Q}_{\alpha}=s y_{i}^{\alpha}+(1-s) Q_{\alpha}$. Then since $s \in[0,1]$ and $Q_{\alpha}$ is convex, $\tilde{Q}_{\alpha} \subset Q_{\alpha}$ and

$$
\int_{Q_{\alpha}}\left|D^{l} \mu\left(z(1-s)+s y_{i}^{\alpha}\right)\right| \mathrm{d} z=\int_{\tilde{Q}_{\alpha}}(1-s)^{N}\left|D^{l} \mu(y)\right| \mathrm{d} y \leq \int_{Q_{\alpha}}\left|D^{l} \mu(y)\right| \mathrm{d} y .
$$

For $0 \leq l \leq k+1$,

$$
\sum_{\alpha \in k \mathbb{Z}^{N},\left|z_{\alpha}\right|>r} \int_{Q_{\alpha}}\left|D^{l} \mu(y)\right| \mathrm{d} y \leq\|\mu\|_{W^{k+1,1}\left(\mathbb{R}^{N} \backslash B_{r}\right)},
$$

so we conclude that for $p \in\{1, \infty\}$,

$$
\left\|\mathcal{L}^{h}[\psi]-\mathcal{L}^{\mu, r}[\psi]\right\|_{L^{p}\left(\mathbb{R}^{N}\right)} \leq C h^{k+1}\|\psi\|_{W^{k+1, p}\left(\mathbb{R}^{N}\right)}\|\mu\|_{W^{k+1,1}\left(\mathbb{R}^{N} \backslash B_{r}\right)} .
$$

The proof is complete.

From Lemmas 4.14, 4.15, and 3.1, we have the following result on admissibility.

Corollary 4.16. Assume $\left(\mathrm{A}_{\mu}\right), 0 \leq k \leq 6,0<r=o_{h}(1), \mathrm{d} \mu(z)=\mu(z) \mathrm{d} z$ for a density $\mu \in C\left(\mathbb{R}^{N} \backslash B_{r}\right) \cap W^{k+1,1}\left(\mathbb{R}^{N} \backslash B_{r}\right)$, and $\mathcal{L}^{\mu, r}$ is defined by (4.12). If $h^{k+1}\|\mu\|_{W^{k+1,1}\left(\mathbb{R}^{N} \backslash B_{r}\right)} \rightarrow 0$ as $h \rightarrow 0$, then the family $\left\{\mathcal{L}^{h}\right\}_{h>0}$ given by (4.16) is an admissible approximation of $\mathcal{L}^{\mu, r}$.

We expect to have more precise results when $\mu$ comes from a fractional differential operator, i.e. a precise estimate on how $\|\mu\|_{W^{k+1,1}\left(\mathbb{R}^{N} \backslash B_{r}\right)}$ depends on $r$. However stronger assumptions than $\left(\mathrm{A}_{\mu_{\alpha}}\right)$ are needed here. It is easy to find such conditions in general, but for simplicity we only focus on the fractional Laplacian case.

Corollary 4.17 (Fractional Laplace). Let $\alpha \in(0,2), 0 \leq h \leq r=o_{h}(1), 0 \leq$ $k \leq 6, \mathcal{L}^{\mu, r}$ be defined by $(4.12)$ with density $\mu(z)=\frac{1}{|z|^{N+\alpha}}$. If $h=o\left(r^{\frac{\alpha+k+1}{k+1}}\right)$, then the family $\left\{\mathcal{L}^{h}\right\}_{h>0}$ given by (4.16) is an admissible approximation of $\mathcal{L}^{\mu, r}$. Moreover, for $p=\{1, \infty\}$,

$$
\left\|\mathcal{L}^{\mu, r}[\psi]-\mathcal{L}^{h}[\psi]\right\|_{L^{p}\left(\mathbb{R}^{N}\right)} \leq C h^{k+1} r^{-\alpha-k-1} .
$$

Proof. Since $D^{k} \mu(z)=O\left(\frac{1}{|z|^{N+\alpha+k}}\right)$ and $\int_{|z|>r} \frac{1}{|z|^{N+\alpha+k}} d z=c \int_{r}^{\infty} \frac{1}{r^{1+\alpha+k}} \mathrm{~d} r=$ $C \frac{1}{r^{\alpha+k}}$, we find that $\|\mu\|_{W^{k+1,1}\left(\mathbb{R}^{N} \backslash B_{r}\right)}=O\left(\frac{1}{r^{\alpha+k+1}}\right)$.

Remark 4.18 (Fractional Laplace). Combining the discretizations of Corollary 4.17 (Newton-Cotes for the nonsingular part) and Lemma 4.5 (adapted vanishing viscosity for the singular part) we get a high order monotone discretization of the fractional Laplacian with (combined) local truncation error,

$$
E=O\left(r^{4-\alpha}+h^{k+1} r^{-\alpha-k-1}\right) .
$$

The optimal choice of $r$ is $r=h^{\frac{k+1}{k+5}}$, which leads to a LTE of $O\left(h^{\frac{k+1}{k+5}(4-\alpha)}\right)$. Note that for all $\alpha \in(0,2)$, this rate is increasing in $k$, superlinear for $k \geq 3$, and superquadratic in the limit $k \rightarrow \infty$. The best choice giving an admissible (monotone) scheme is $k=6$ and $r=O\left(h^{\frac{7}{11}}\right)$ with a LTE of $O\left(h^{\frac{7}{11}(4-\alpha)}\right)$.

Remark 4.19 (Random walk approximation). In [65, 12] the Fractional Heat Equation is formally derived from a random walk approximation with arbitrarily long jumps,

$$
U^{j}\left(x_{\beta}\right)=U^{j-1}\left(x_{\beta}\right)+\Delta t \sum_{\gamma \in \mathbb{Z}^{N} \backslash\{0\}}\left(U^{j-1}\left(x_{\beta}+z_{\gamma}\right)-U^{j-1}\left(x_{\beta}\right)\right) \omega_{\gamma, h},
$$

where $\omega_{\gamma, h}:=\mu^{\alpha}\left(z_{\gamma}\right) h^{N}, \mu^{\alpha}(z):=\frac{c_{N, \alpha}}{|z|^{N+\alpha}}$ for $z \neq 0, \mu^{\alpha}(0)=0$, and $c_{N, \alpha}$ is such that $\sum_{\gamma \in \mathbb{Z}^{N}} \mu^{\alpha}(\gamma)=1$. Note that here $\Delta t=h^{\alpha}$ and $\frac{\mu^{\alpha}(\gamma)}{h^{\alpha}}=h^{N} \mu^{\alpha}\left(z_{\gamma}\right)$. The 
scheme corresponds to the spatial discretization (4.16) with $k=0$, and converges by Corollary 4.17 and Theorem 3.6 if we require $\left|z_{\gamma}\right|>r=c h^{\frac{1}{\alpha+1+\epsilon}}$ for some $c, \epsilon>0$.

4.5. A second order discretization of the fractional Laplacian. The fractional Laplacian $-(-\Delta)^{\frac{\alpha}{2}}$ can be discretized by the corresponding power of the discrete Laplacian

$$
\Delta_{h} \psi(x)=\frac{1}{h^{2}} \sum_{i=1}^{N} \psi\left(x+e_{i} h\right)+\psi\left(x-e_{i} h\right)-2 \psi(x)
$$

defined via subordination as

$$
\left(-\Delta_{h}\right)^{\frac{\alpha}{2}}[\psi](x):=\frac{1}{\Gamma\left(-\frac{\alpha}{2}\right)} \int_{0}^{\infty}\left(\mathrm{e}^{t \Delta_{h}} \psi(x)-\psi(x)\right) \frac{\mathrm{d} t}{t^{1+\frac{\alpha}{2}}},
$$

where $\Psi(x, t):=\mathrm{e}^{t \Delta_{h}} \psi(x)$ is the solution of the semi-discrete heat equation

$$
\left\{\begin{array}{lll}
\partial_{t} \Psi=\Delta_{h} \Psi & \text { for } & (x, t) \in \mathbb{R}^{N} \times(0, \infty), \\
\Psi(x, 0)=\psi(x) & \text { for } & x \in \mathbb{R}^{N} .
\end{array}\right.
$$

The solution of (4.18) has an explicit representation formula,

$$
\Psi(x, t):=\mathrm{e}^{t \Delta_{h}} \psi(x)=\sum_{\beta} \psi\left(x-z_{\beta}\right) G\left(\beta, \frac{t}{h^{2}}\right),
$$

where $G(\beta, t)=\mathrm{e}^{-2 N t} \prod_{i=1}^{N} I_{\left|\beta_{i}\right|}(2 t)$ is the fundamental solution of (4.18) and $I_{m}$ the modified Bessel function of first kind and order $m \in \mathbb{N}$. Moreover $G \geq 0$, $G(\beta, t)=G(-\beta, t)$, and $\sum_{\beta} G(\beta, t)=1$, see e.g. $[20,56]$.

The original idea of this discretization is due to Ciaurri et al. [20, 21]. Here the authors obtain a Local Truncation Errors of $O\left(h^{2-\alpha}\right)$ for $N=1$. These results were then extended to $N>1$ in $[28,27]$ for various boundary value problems and the whole space case. Here the authors also improve the local truncation error to $O\left(h^{2}\right)$ independently of $\alpha \in(0,2)$. This means that the approximation $\left(-\Delta_{h}\right)^{\frac{\alpha}{2}}$ preserves the $O\left(h^{2}\right)$ error bound of the discrete Laplacian $\Delta_{h}$.

We now show that (4.17) is an admissible operator. First we express (4.17) in the form (FD). This result has essentially been proved in [20].

Lemma 4.20. Let $h>0, \alpha \in(0,2)$, and $N \geq 1$. Then $\left(-\Delta_{h}\right)^{\frac{\alpha}{2}}$ given by (4.17) is an operator of the form (FD),

$$
\left(-\Delta_{h}\right)^{\frac{\alpha}{2}}[\psi](x)=\sum_{\beta \neq 0}\left(\psi\left(x+z_{\beta}\right)-\psi(x)\right) K_{\beta, h},
$$

where $K_{\beta, h}=\frac{1}{h^{\alpha}} \frac{1}{\Gamma\left(-\frac{\alpha}{2}\right)} \int_{0}^{\infty} G(\beta, t) \frac{\mathrm{d} t}{t^{1+\frac{\alpha}{2}}}$.

Proof. By (4.17), the representation formula for $\Psi$, and $\sum_{\beta} G\left(\beta, \frac{t}{h^{2}}\right)=1$,

$$
\begin{aligned}
\left(-\Delta_{h}\right)^{\frac{\alpha}{2}}[\psi](x) & :=\frac{1}{\Gamma\left(-\frac{\alpha}{2}\right)} \int_{0}^{\infty}\left(\sum_{\beta} \psi\left(x-z_{\beta}\right) G\left(\beta, \frac{t}{h^{2}}\right)-\psi(x)\right) \frac{\mathrm{d} t}{t^{1+\frac{\alpha}{2}}} \\
& =\frac{1}{\Gamma\left(-\frac{\alpha}{2}\right)} \int_{0}^{\infty} \sum_{\beta}\left(\psi\left(x-z_{\beta}\right)-\psi(x)\right) G\left(\beta, \frac{t}{h^{2}}\right) \frac{\mathrm{d} t}{t^{1+\frac{\alpha}{2}}} \\
& =\sum_{\beta}\left(\psi\left(x-z_{\beta}\right)-\psi(x)\right) \frac{1}{\Gamma\left(-\frac{\alpha}{2}\right)} \int_{0}^{\infty} G\left(\beta, \frac{t}{h^{2}}\right) \frac{\mathrm{d} t}{t^{1+\frac{\alpha}{2}}} .
\end{aligned}
$$

The change of variables $\tau=t / h^{2}$ finishes the proof. 
Remark 4.21. In dimension $N=1$ a more explicit expression for $K_{\beta, h}$ is given in [21]: $K_{j, h}:=\frac{1}{h^{\alpha}} \frac{2^{\alpha} \Gamma\left(\frac{1+\alpha}{2}\right) \Gamma\left(|j|-\frac{\alpha}{2}\right)}{\sqrt{\pi}\left|\Gamma\left(-\frac{\alpha}{2}\right)\right| \Gamma\left(|j|+1+\frac{\alpha}{2}\right)}$ for $j \in \mathbb{Z}, j \neq 0$.

Lemma 4.22. Assume $h>0, \alpha \in(0,2)$, and $N \geq 1$. The family $\left\{-\left(-\Delta_{h}\right)^{\frac{\alpha}{2}}\right\}_{h>0}$ given by (4.17) (or (4.19)) is an admissible approximation of $-(-\Delta)^{\frac{\alpha}{2}}$ with Local Truncation Error

$$
\left\|\left(-\Delta_{h}\right)^{\frac{\alpha}{2}}[\psi]-(-\Delta)^{\frac{\alpha}{2}}[\psi]\right\|_{L^{p}(\mathbb{R})}=O\left(h^{2}\right),
$$

for $p=\{1, \infty\}$ and $\psi \in C_{c}^{\infty}\left(\mathbb{R}^{N}\right)$.

Proof. By Lemma 4.20, $-\left(-\Delta_{h}\right)^{\frac{\alpha}{2}}$ is an operator in the form (FD) explicitly given by (4.19). We show that it is in the class $\left(\mathrm{A}_{\nu}\right)$. It is clear that $K_{\beta, h}$ is nonnegative and symmetric in $\beta$ since these properties are shared by $G$. Next,

$$
C_{2}:=\sum_{\beta \neq 0} \int_{1}^{\infty} G(\beta, t) \frac{\mathrm{d} t}{t^{1+\frac{\alpha}{2}}}=\int_{1}^{\infty} \sum_{\beta \neq 0} G(\beta, t) \frac{\mathrm{d} t}{t^{1+\frac{\alpha}{2}}} \leq \int_{1}^{\infty} \frac{\mathrm{d} t}{t^{1+\frac{\alpha}{2}}}=\frac{2}{\alpha},
$$

and

$$
C_{1}:=\sum_{\beta \neq 0} \int_{0}^{1} G(\beta, t) \frac{\mathrm{d} t}{t^{1+\frac{\alpha}{2}}}=\int_{0}^{1} \sum_{\beta \neq 0} G(\beta, t) \frac{\mathrm{d} t}{t^{1+\frac{\alpha}{2}}}=\int_{0}^{1}(1-G(0, t)) \frac{\mathrm{d} t}{t^{1+\frac{\alpha}{2}}} .
$$

By regularity and the properties of $G,|1-G(0, t)|=|G(0,0)-G(0, t)| \leq C t$ for $C=\max _{\xi \in[0, t]}\left\{\partial_{t} G(0, t)\right\}$, and then $C_{1} \leq C \int_{0}^{1} t \frac{\mathrm{d} t}{t^{1+\frac{\alpha}{2}}}=C \frac{2}{2-\alpha}$. We conclude that

$$
\sum_{\beta \neq 0} K_{\beta, h}=\frac{1}{h^{\alpha}} \frac{1}{\Gamma\left(-\frac{\alpha}{2}\right)}\left(C_{1}+C_{2}\right)<+\infty
$$

and that $-\left(-\Delta_{h}\right)^{\frac{\alpha}{2}}$ is in the class $\left(\mathrm{A}_{\nu}\right)$ by Lemma 3.1 .

Now we need to show that the discretization is consistent. We proceed as in $[28,27]$. Using directly the semigroup formulation (4.17), we get that

$$
\left(-\Delta_{h}\right)^{\frac{\alpha}{2}}[\psi](x)-(-\Delta)^{\frac{\alpha}{2}}[\psi](x)=\frac{1}{\Gamma\left(-\frac{\alpha}{2}\right)} \int_{0}^{\infty}\left(\mathrm{e}^{t \Delta_{h}} \psi(x)-\mathrm{e}^{t \Delta} \psi(x)\right) \frac{\mathrm{d} t}{t^{1+\frac{\alpha}{2}}}
$$

where $\mathrm{e}^{t \Delta} \psi(x)$ is the solution of the heat equation with initial condition $\psi$. Assume for the moment that the following estimate holds for $p=\{1, \infty\}$ :

$$
\left\|\mathrm{e}^{t \Delta_{h}} \psi(\cdot)-\mathrm{e}^{t \Delta} \psi(\cdot)\right\|_{L^{p}\left(\mathbb{R}^{N}\right)} \leq\left\{\begin{array}{l}
C t h^{2} \quad \text { for } \quad 0 \leq t \leq 1, \\
C \frac{h^{2}}{t} \quad \text { for } t \geq 1,
\end{array}\right.
$$

for some $C>0$ depending on $\psi$. Then,

$$
\begin{aligned}
\left\|\left(-\Delta_{h}\right)^{\frac{\alpha}{2}}[\psi]-(-\Delta)^{\frac{\alpha}{2}}[\psi]\right\|_{L^{p}\left(\mathbb{R}^{N}\right)} & \leq \frac{1}{\Gamma\left(-\frac{\alpha}{2}\right)} \int_{0}^{\infty}\left\|\mathrm{e}^{t \Delta_{h}} \psi(\cdot)-\mathrm{e}^{t \Delta} \psi(\cdot)\right\|_{L^{p}\left(\mathbb{R}^{N}\right)} \frac{\mathrm{d} t}{t^{1+\frac{\alpha}{2}}} \\
& \leq C h^{2} \int_{0}^{1} \frac{\mathrm{d} t}{t^{\frac{\alpha}{2}}}+C h^{2} \int_{1}^{\infty} \frac{\mathrm{d} t}{t^{2+\frac{\alpha}{2}}} \leq \tilde{C} h^{2} .
\end{aligned}
$$

From the $L^{\infty}$-estimate and Lemma 3.2, we also have that (UL) holds.

It only remains to prove estimate (4.20). Recall that

$$
\mathrm{e}^{t \Delta} \psi(x)=\int_{\mathbb{R}^{N}} \psi(x-y) F(y, t) \mathrm{d} y \quad \text { with } \quad F(x, t)=\frac{1}{(4 \pi t)^{N / 2}} \mathrm{e}^{-\frac{|x|^{2}}{4 t}},
$$


and set $\tau(x, t):=\partial_{t} \mathrm{e}^{t \Delta} \psi(x)-\Delta_{h} \mathrm{e}^{t \Delta} \psi(x)$. Since $\mathrm{e}^{t \Delta} \psi$ is smooth, a Taylor expansion argument and the properties of $F, D^{4} F$ show that

$$
\|\tau(\cdot, t)\|_{L^{p}} \leq h^{2}\left\|D^{4} e^{t \Delta} \psi\right\|_{L^{p}} \leq\left\{\begin{array}{l}
h^{2}\left\|D^{4} \psi\right\|_{L^{p}}\|F\|_{L^{1}} \leq C h^{2} \quad \text { for } \quad 0 \leq t \leq 1, \\
h^{2}\|\psi\|_{L^{p}}\left\|D^{4} F\right\|_{L^{1}} \leq C \frac{h^{2}}{t^{2}} \quad \text { for } \quad t \geq 1 .
\end{array}\right.
$$

Now let $E(x, t):=\mathrm{e}^{t \Delta_{h}} \psi(x)-\mathrm{e}^{t \Delta} \psi(x)$ and note that $\partial_{t} E(x, t)=\Delta_{h} E(x, t)+\tau(x, t)$ and $E(x, 0)=0$. The weak maximum principle (for (4.18)) and a standard $L^{1}$ bound then immediately yield

$$
\|E(\cdot, t)\|_{L^{p}} \leq \int_{0}^{t}\|\tau(\cdot, s)\|_{L^{p}} \mathrm{~d} s
$$

and (4.20) follows from (4.21).

4.6. Approximation of the nonlinearity. As we saw in Section 3.3, we need to impose the condition (CFL) when the schemes have some explicit part (i.e. when $0 \leq \theta<1$ ). This condition requires the nonlinearity $\varphi$ to be (locally) Lipschitz. But our results can handle merely continuous $\varphi$. If $\varphi$ is not locally Lipschitz as e.g. in the fast diffusion case, we must replace it by a Lipschitz approximation to get explicit monotone schemes. To be precise, we approximate $\varphi$ by a sequence of nondecreasing Lipschitz functions $\varphi^{\epsilon}$ converging locally uniformly as $\epsilon=o_{h}(1) \rightarrow 0^{+}$. The (CFL) condition is then

$$
\Delta t(1-\theta) L_{\varphi^{\epsilon}} \nu_{h}\left(\mathbb{R}^{N}\right) \leq 1
$$

Note that $L_{\varphi^{\epsilon}} \rightarrow \infty$ as $\epsilon \rightarrow 0^{+}$making (CFL) a more and more restrictive condition as $h$ approaches zero.

There are several ways of choosing the nonlinearity $\varphi^{\epsilon}$ in an admissible way. Two simple and general choices (cf. e.g. [36, 66]) are

$$
\varphi^{\epsilon}(\xi):=\left(\varphi * \omega_{\epsilon}\right)(\xi)-\left(\varphi * \omega_{\epsilon}\right)(0) \quad \text { and } \quad \varphi^{\epsilon}(\xi)+\epsilon \xi
$$

where $\omega_{\epsilon}$ is a standard mollifier in $\mathbb{R}$. However, in many applications $\varphi$ is nonLipschitz only at the origin. A well-known example is the Fast Diffusion Equation where $\varphi(\xi)=\xi^{m}$ for $0<m<1$. In this case an easier and more efficient choice is

$$
\varphi^{\epsilon}(\xi)= \begin{cases}\varphi(\xi+\epsilon)-\varphi(\epsilon) & \text { if } \quad \xi \geq 0 \\ \varphi(\xi-\epsilon)-\varphi(-\epsilon) & \text { if } \quad \xi<0 .\end{cases}
$$

Clearly $\varphi^{\epsilon} \rightarrow \varphi$ locally uniformly and $\varphi^{\epsilon}$ is Lipschitz with Lipschitz constant

$$
L_{\varphi^{\epsilon}}=\left|\left(\varphi^{\epsilon}\right)^{\prime}(0)\right|=\frac{m}{\epsilon^{1-m}} .
$$

Moreover, this approximation enjoys the very interesting property of preserving the zero level sets of the solution since $\varphi^{\epsilon}(0)=0=\varphi(0)$.

\section{NUMERICAL EXPERIMENTS IN 1D}

In this section, we test our numerical schemes on interesting special cases of (1.1)-(1.2) in one space dimension that involve the fractional Laplacian,

$$
\partial_{t} u(x, t)+(-\Delta)^{\frac{\alpha}{2}}[\varphi(u(\cdot, t))](x)=g(x, t) \quad \text { in } \quad Q_{T}:=\mathbb{R} \times(0, T)
$$

for $\alpha \in(0,2)$. All the schemes are of the form (3.1), and since our initial data and right-hand sides will be smooth, we simply take $U_{\beta}^{0}=u_{0}\left(x_{\beta}\right)$ and $F_{\beta}^{j}=f\left(x_{\beta}, t_{j}\right)$. We consider explicit $(\theta=0)$ and implicit $(\theta=1)$ schemes and the following spatial discretizations of the nonlocal operators:

1) $\mathrm{MpR}=$ Midpoint Rule + trivial discretization for singular part 
2) $\mathrm{FOI}=$ First Order Interpolation + trivial discretization for singular part

3) $\mathrm{SOI}=$ Second Order Interpolation + adapted vanishing viscosity

4) $\mathrm{PDL}=$ Powers of the Discrete Laplacian

All experiments have been run on equidistant grids in space and time, with $\Delta t$ such that (CFL) holds and the overall order of convergence is determined by the spatial discretization. To compute the solutions we restrict to a (sufficiently) large bounded spatial domain $I$ and set the numerical solution equal zero outside. See Section 5.5 for some numerical tests on how the size of the domain affects the error.

The error is calculated either in $L^{1}(I)$ or $L^{\infty}(I)$ at a certain time $T>0$. To compute the error, we consider examples with (i) known exact solutions, or (ii) we force a nice function $v$ to be a solution by taking

$$
g(x, t)=\partial_{t} v(x, t)+(-\Delta)^{\frac{\alpha}{2}}[\varphi(v(\cdot, t))](x),
$$

or (iii) we compute the errors numerically. In the latter case we assume the error $E$ satisfies $E=C h^{\gamma}$, take $h_{j}=c 2^{-j}$, and compute an estimate of the rate $\gamma$ as

$$
\gamma=\log _{2}\left(\frac{E_{j-1}}{E_{j}}\right) .
$$

5.1. Fractional Heat Equation (explicit scheme). We consider (5.1) with $\varphi(\xi)=\xi, \alpha=1$, and $g \equiv 0$. This is a Fractional Heat Equation with explicit fundamental solution $K(x, t)=\frac{t}{t^{2}+|x|^{2}}$. We take $u_{0}(x)=K(x, 1)$ so that the exact solution is

$$
u(x, t)=K(x, t+1) .
$$

We also take $I=[-5000,5000]$. By (5.3), $u \sim 10^{-8}$ outside of $I$, and hence is negligible in the error analysis. In Figure 1 and Table 1 we show the error and rates at time $T=1$.

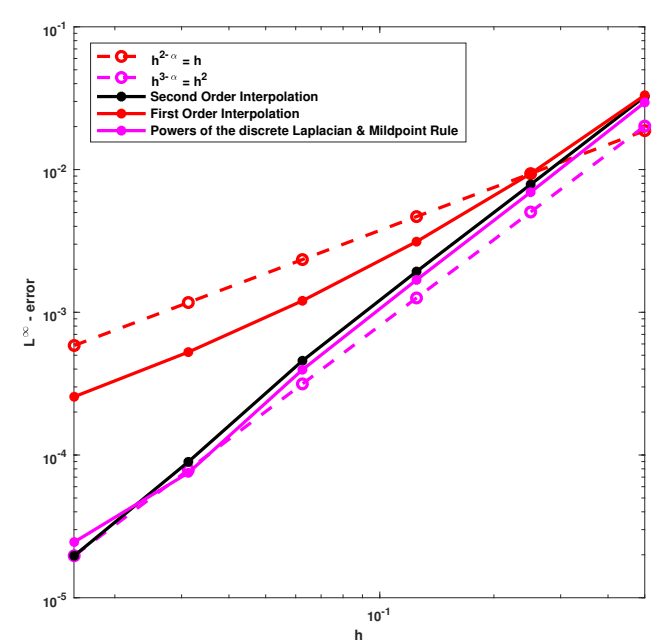

FIGURE 1. $L^{\infty}$-error at $T=1$ with $I=[-5000,5000]$ for the exact solution (5.3) of (5.1) with $\alpha=1, \varphi(\xi)=\xi$ and $g \equiv 0$.

Conclusion: The Midpoint Rule and the Powers of the Discrete Laplacian coincide when $\alpha=1$ and $N=1$ (see Section 4). The theoretical convergence rates are confirmed for all tested methods. Note that when $h=1.56 \mathrm{e}-2$, the rate for MpR and PDL is $\gamma=1.61$ in stead of $\gamma \sim 2$. This is a consequence of how the rates are calculated since the previous value $\gamma=2.40$ is much better than predicted. If we calculate the rate for $h=1.56 \mathrm{e}-2$ with respect to $h=6.25 \mathrm{e}-2$, we get $\gamma=2.01$. 


\begin{tabular}{l|l|l|l|l|l|l|l|l}
\multicolumn{2}{l}{$h$} & MpR & \multicolumn{2}{l}{ FOI } & SOI & \multicolumn{2}{l}{ PDL } \\
\cline { 2 - 8 } & error & $\gamma$ & error & $\gamma$ & error & $\gamma$ & error & $\gamma$ \\
\hline $5.00 \mathrm{e}-1$ & $2.95 \mathrm{e}-2$ & & $3.31 \mathrm{e}-2$ & & $3.24 \mathrm{e}-3$ & & $2.95 \mathrm{e}-2$ & \\
$2.50 \mathrm{e}-1$ & $6.94 \mathrm{e}-3$ & 2.08 & $9.40 \mathrm{e}-3$ & 1.82 & $7.89 \mathrm{e}-3$ & 2.04 & $6.94 \mathrm{e}-3$ & 2.08 \\
$1.25 \mathrm{e}-1$ & $1.68 \mathrm{e}-3$ & 2.04 & $3.12 \mathrm{e}-3$ & 1.58 & $1.93 \mathrm{e}-3$ & 2.03 & $1.68 \mathrm{e}-3$ & 2.04 \\
$6.25 \mathrm{e}-2$ & $3.95 \mathrm{e}-4$ & 2.09 & $1.20 \mathrm{e}-4$ & 1.37 & $4.57 \mathrm{e}-4$ & 2.08 & $3.95 \mathrm{e}-4$ & 2.09 \\
$3.13 \mathrm{e}-2$ & $7.50 \mathrm{e}-5$ & 2.40 & $5.26 \mathrm{e}-4$ & 1.19 & $8.96 \mathrm{e}-5$ & 2.35 & $7.50 \mathrm{e}-5$ & 2.40 \\
$1.56 \mathrm{e}-2$ & $2.45 \mathrm{e}-5$ & 1.61 & $2.56 \mathrm{e}-4$ & 1.03 & $1.97 \mathrm{e}-5$ & 2.18 & $2.45 \mathrm{e}-5$ & 1.61
\end{tabular}

TABLE $1 . L^{\infty}$-error at $T=1$ with $I=[-5000,5000]$ for the exact solution (5.3) of (5.1) with $\alpha=1, \varphi(\xi)=\xi$ and $g \equiv 0$.

5.2. Fractional Porous Medium Equation (explicit scheme). We consider (5.1) with $\varphi(\xi)=\xi^{2}$. An explicit solution of that problem is known when $\alpha=\frac{1}{3}$ (cf. [49]), but its slow decay at infinity makes it difficult to find a reasonably small computational domain $I$. To overcome this issue we impose $v=(t+1) \mathrm{e}^{-|x|^{2}}$ as a solution by taking $g$ according to (5.2). Note that now the solution has exponential decay. The errors and rates of convergence are shown for $I=[-100,100]$ at time $T=1$ for $\alpha=0.5$ (resp. $\alpha=1.5$ ) in Figure 2 and Table 2 (resp. Table 3).
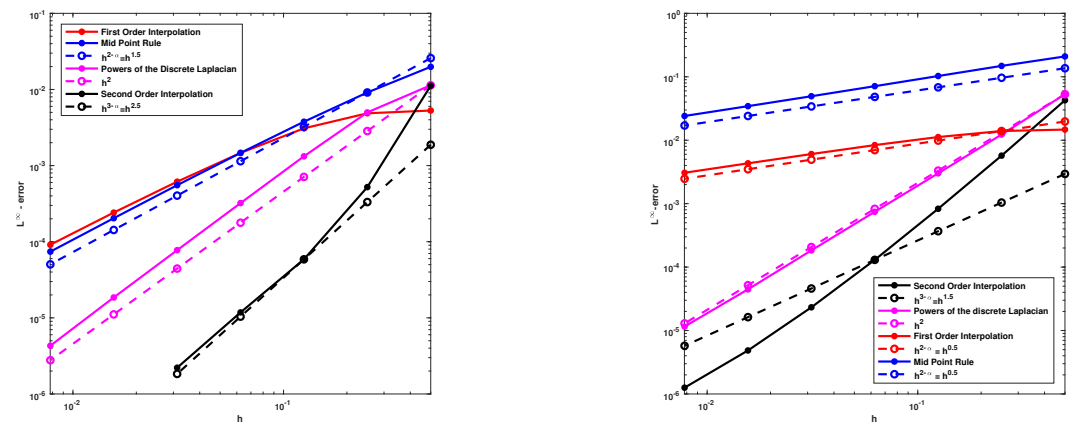

FiguRE 2. $L^{\infty}$-error at $T=1$ with $I=[-100,100]$ for $(5.1)$ with $\varphi(\xi)=\xi^{2}$ and $g$ by (5.2) with $v(x, t)=(t+1) \mathrm{e}^{-|x|^{2}}$. Left: $\alpha=0.5$. Right: $\alpha=1.5$.

\begin{tabular}{l|l|l|l|l|l|l|l|l}
\multirow{2}{*}{$h$} & \multicolumn{2}{l}{ MPR } & \multicolumn{2}{l}{ FOI } & \multicolumn{2}{l}{ SOI } & \multicolumn{2}{l}{ PDL } \\
\cline { 2 - 9 } & error & $\gamma$ & error & $\gamma$ & error & $\gamma$ & error & $\gamma$ \\
\hline $5.00 \mathrm{e}-1$ & $1.98 \mathrm{e}-2$ & & $5.29 \mathrm{e}-3$ & & $1.12 \mathrm{e}-2$ & & $1.14 \mathrm{e}-2$ & \\
$2.50 \mathrm{e}-1$ & $9.13 \mathrm{e}-3$ & 1.22 & $4.86 \mathrm{e}-3$ & 0.12 & $5.21 \mathrm{e}-4$ & 4.42 & $5.00 \mathrm{e}-3$ & 1.19 \\
$1.25 \mathrm{e}-1$ & $3.77 \mathrm{e}-3$ & 1.28 & $3.09 \mathrm{e}-3$ & 0.65 & $5.87 \mathrm{e}-5$ & 3.15 & $1.32 \mathrm{e}-3$ & 1.91 \\
$6.25 \mathrm{e}-2$ & $1.47 \mathrm{e}-3$ & 1.36 & $1.47 \mathrm{e}-3$ & 1.07 & $1.18 \mathrm{e}-5$ & 2.31 & $3.21 \mathrm{e}-4$ & 2.04 \\
$3.13 \mathrm{e}-2$ & $5.53 \mathrm{e}-4$ & 1.40 & $6.15 \mathrm{e}-4$ & 1.25 & $2.21 \mathrm{e}-6$ & 2.41 & $7.74 \mathrm{e}-5$ & 2.05 \\
$1.56 \mathrm{e}-2$ & $2.04 \mathrm{e}-4$ & 1.44 & $2.41 \mathrm{e}-4$ & 1.35 & - & - & $1.85 \mathrm{e}-5$ & 2.06 \\
$7.81 \mathrm{e}-3$ & $7.41 \mathrm{e}-5$ & 1.46 & $9.13 \mathrm{e}-5$ & 1.40 & - & - & $4.30 \mathrm{e}-6$ & 2.11
\end{tabular}

TABLE 2. $L^{\infty}$-error at $T=1$ with $I=[-100,100]$ for $(5.1)$ with $\alpha=0.5$, $\varphi(\xi)=\xi^{2}$ and $g$ by (5.2) with $v(x, t)=(t+1) \mathrm{e}^{-|x|^{2}}$.

Conclusion: When $\alpha=0.5$ all the expected rates are recovered. For the Second Order Interpolation, we excluded the last two rows because the error was much smaller compared to the other methods - see Table 2. It is also worth noting that when $\alpha=1.5$ - see Table 3, the expected rate of convergence for SOI is $\gamma=1.5$ while the rate obtained by the experiment is $\sim 2.0$. 


\begin{tabular}{l|l|l|l|l|l|l|l|l}
\multicolumn{2}{l}{$h$} & \multicolumn{2}{l}{ MPR } & \multicolumn{2}{l}{ FOI } & \multicolumn{2}{l}{ SOI } & \multicolumn{2}{l}{ PDL } \\
\cline { 2 - 8 } & error & $\gamma$ & error & $\gamma$ & error & $\gamma$ & error & $\gamma$ \\
\hline $5.00 \mathrm{e}-1$ & $2.10 \mathrm{e}-1$ & & $1.47 \mathrm{e}-2$ & & $4.26 \mathrm{e}-2$ & & $5.30 \mathrm{e}-2$ & \\
$2.50 \mathrm{e}-1$ & $1.49 \mathrm{e}-1$ & 0.49 & $1.40 \mathrm{e}-2$ & 0.08 & $5.71 \mathrm{e}-3$ & 2.90 & $1.23 \mathrm{e}-2$ & 2.11 \\
$1.25 \mathrm{e}-1$ & $1.03 \mathrm{e}-1$ & 0.53 & $1.12 \mathrm{e}-2$ & 0.31 & $8.30 \mathrm{e}-4$ & 2.78 & $3.01 \mathrm{e}-3$ & 2.03 \\
$6.25 \mathrm{e}-2$ & $7.11 \mathrm{e}-2$ & 0.53 & $8.37 \mathrm{e}-3$ & 0.42 & $1.30 \mathrm{e}-4$ & 2.67 & $7.44 \mathrm{e}-4$ & 2.16 \\
$3.13 \mathrm{e}-2$ & $4.93 \mathrm{e}-2$ & 0.53 & $6.05 \mathrm{e}-3$ & 0.47 & $2.32 \mathrm{e}-5$ & 2.48 & $1.83 \mathrm{e}-4$ & 2.02 \\
$1.56 \mathrm{e}-2$ & $3.44 \mathrm{e}-2$ & 0.52 & $4.32 \mathrm{e}-3$ & 0.49 & $4.85 \mathrm{e}-6$ & 2.25 & $4.46 \mathrm{e}-5$ & 2.04 \\
$7.81 \mathrm{e}-3$ & $2.41 \mathrm{e}-2$ & 0.51 & $3.07 \mathrm{e}-3$ & 0.49 & $1.25 \mathrm{e}-6$ & 1.94 & $1.16 \mathrm{e}-5$ & 1.95
\end{tabular}

TABLE 3. $L^{\infty}$-error at $T=1$ with $I=[-100,100]$ for $(5.1)$ with $\alpha=1.5$, $\varphi(\xi)=\xi^{2}$ and $g$ by $(5.2)$ with $v(x, t)=(t+1) \mathrm{e}^{-|x|^{2}}$.

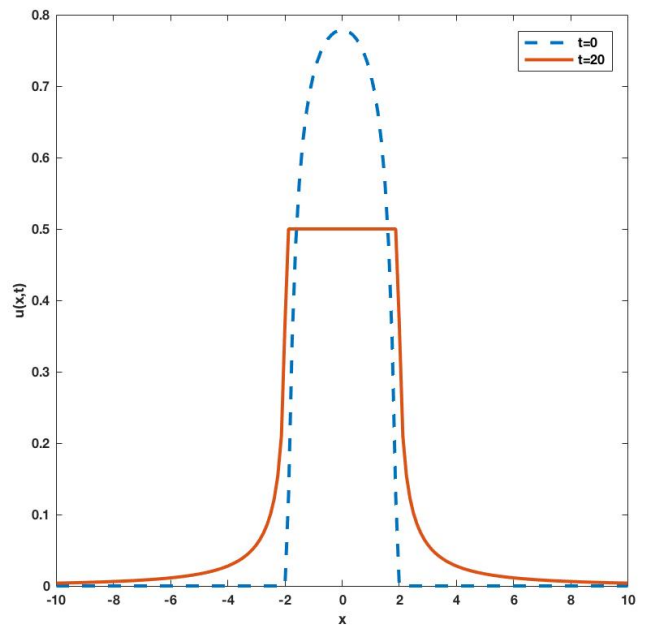

FIGURE 3. Solution of (5.1) for $\alpha=1, \varphi(\xi)=\max \{0, \xi-0.5\}$, and $g \equiv 0$.

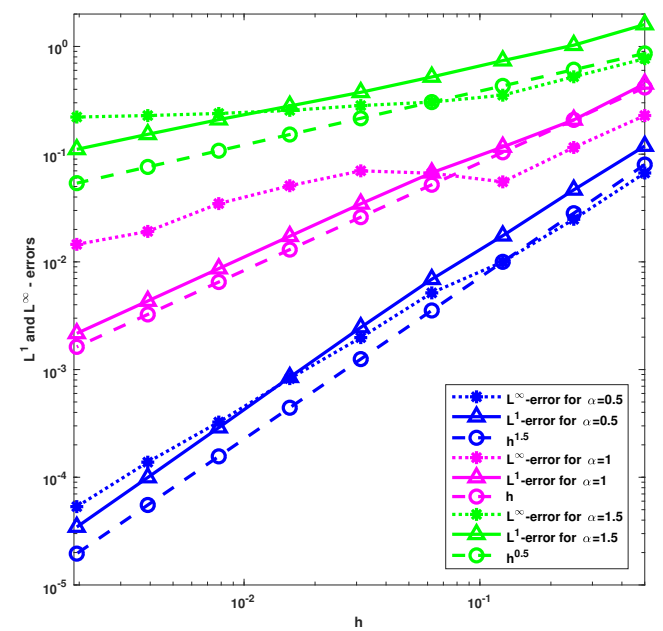

FIGURE 4. $L^{\infty}$ - and $L^{1}$-errors with $\mathrm{MpR}$ at $T=1$ with $I=[-100,100]$ for (5.1) with $\varphi$ given by (5.4) and $g$ by (5.2) with $v(x, t)=(t+1) \mathrm{e}^{-|x|^{2}}$. 
5.3. Stefan type problem (explicit scheme). First we consider (5.1) with $\varphi(\xi)=\max \{0, \xi-0.5\}$ (a globally Lipschitz function) and $g \equiv 0$. Solutions of such problems can loose regularity as we see in Figure 3 (above). Here we have used $C_{\mathrm{c}}^{\infty}(\mathbb{R})$ initial data $u_{0}(x)=\mathrm{e}^{-\frac{1}{4-x^{2}}} \mathbf{1}_{[-2,2]}(x)$.

Next we consider (5.1) with

$$
\varphi(\xi)= \begin{cases}\xi & \text { if } \xi<0.2 \\ 0.2 & \text { if } \quad 0.2 \leq \xi<0.4 \\ \xi-0.2 & \text { if } \xi \geq 0.4\end{cases}
$$

and impose $v(x, t)=(t+1) \mathrm{e}^{-|x|^{2}}$ as the solution by taking $g$ as in (5.2). We run experiments for $\alpha=0.5, \alpha=1$, and $\alpha=1.5$ up to $T=1$ with $I=[-100,100]$ using the Midpoint Rule. The results are given in Figure 4 (above) and Table 4 (below).

Conclusion: It is interesting to note that even when the solution $u \in C^{\infty}\left(\mathbb{R}^{N}\right)$, $\varphi(u)$ is just a Lipschitz function. Therefore $-(-\Delta)^{\frac{1}{2}}[\varphi(u)]$ and $-(-\Delta)^{\frac{3}{4}}[\varphi(u)]$ do not exist in every point and $u$ is not a classical solution. This fact has a strong effect on the convergence rates in $L^{\infty}$, but not in $L^{1}$, see Figure 4 and Table 4.

Remark 5.1. In [10] the authors study a nonlocal Stefan problem of the form (1.1) with $\sigma \equiv 0$ and $\mu$ having a nonnegative continuous compactly supported density $\left(\left(\mathrm{A}_{\mu}\right)\right.$ holds $)$. This case is easier than the above example since $\mathfrak{L}^{\sigma, \mu}$ is bounded. Nonetheless it has discontinuous solutions that are computed numerically in Chapter 7 of [10]. Our results provide a rigorous justification for these computations.

\begin{tabular}{l|l|l|l|l|l|l|l|l|l|l|l|l|}
\multicolumn{1}{l}{$h$} & $\alpha=\frac{1}{2}$ & \multicolumn{1}{l}{$\alpha=1$} & \multicolumn{1}{l}{$\alpha=\frac{3}{2}$} \\
\cline { 2 - 12 } & $L^{\infty}$ & $\gamma$ & $L^{1}$ & $\gamma$ & $L^{\infty}$ & $\gamma$ & $L^{1}$ & $\gamma$ & $L^{\infty}$ & $\gamma$ & $L^{1}$ & $\gamma$ \\
\hline $5.0 \mathrm{e}-1$ & $6.7 \mathrm{e}-2$ & & $1.3 \mathrm{e}-1$ & & $2.3 \mathrm{e}-1$ & & $4.5 \mathrm{e}-1$ & & $7.7 \mathrm{e}-1$ & & $1.6 \mathrm{e}-0$ & \\
$2.5 \mathrm{e}-1$ & $2.5 \mathrm{e}-2$ & 1.4 & $4.7 \mathrm{e}-2$ & 1.4 & $1.2 \mathrm{e}-1$ & 1 & $2.1 \mathrm{e}-1$ & 1.1 & $5.2 \mathrm{e}-1$ & 0.6 & $1.0 \mathrm{e}-0$ & 0.6 \\
$1.3 \mathrm{e}-1$ & $1.0 \mathrm{e}-2$ & 1.3 & $1.8 \mathrm{e}-2$ & 1.4 & $5.5 \mathrm{e}-2$ & 1.1 & $1.2 \mathrm{e}-1$ & 0.8 & $3.5 \mathrm{e}-1$ & 0.6 & $7.4 \mathrm{e}-1$ & 0.5 \\
$6.3 \mathrm{e}-2$ & $5.2 \mathrm{e}-3$ & 1.0 & $6.9 \mathrm{e}-3$ & 1.3 & $6.7 \mathrm{e}-2$ & $<0$ & $6.7 \mathrm{e}-2$ & 0.8 & $3.0 \mathrm{e}-1$ & 0.2 & $5.2 \mathrm{e}-1$ & 0.5 \\
$3.1 \mathrm{e}-2$ & $2.0 \mathrm{e}-3$ & 1.4 & $2.5 \mathrm{e}-3$ & 1.5 & $7.0 \mathrm{e}-2$ & $<0$ & $3.5 \mathrm{e}-2$ & 1.0 & $2.8 \mathrm{e}-1$ & 0.1 & $3.8 \mathrm{e}-1$ & 0.5 \\
$1.6 \mathrm{e}-2$ & $8.2 \mathrm{e}-4$ & 1.3 & $8.5 \mathrm{e}-4$ & 1.5 & $5.1 \mathrm{e}-2$ & 0.5 & $1.7 \mathrm{e}-2$ & 1.0 & $2.6 \mathrm{e}-1$ & 0.2 & $2.8 \mathrm{e}-1$ & 0.4 \\
$7.8 \mathrm{e}-3$ & $3.3 \mathrm{e}-4$ & 1.3 & $2.9 \mathrm{e}-4$ & 1.6 & $3.5 \mathrm{e}-2$ & 0.6 & $8.5 \mathrm{e}-3$ & 1.0 & $2.4 \mathrm{e}-1$ & 0.1 & $2.1 \mathrm{e}-1$ & 0.4 \\
$3.9 \mathrm{e}-3$ & $1.4 \mathrm{e}-4$ & 1.2 & $9.9 \mathrm{e}-5$ & 1.5 & $1.9 \mathrm{e}-2$ & 0.9 & $4.3 \mathrm{e}-3$ & 1.0 & $2.3 \mathrm{e}-1$ & 0.1 & $1.5 \mathrm{e}-1$ & 0.5 \\
$2.0 \mathrm{e}-3$ & $5.3 \mathrm{e}-5$ & 1.4 & $3.4 \mathrm{e}-5$ & 1.5 & $1.5 \mathrm{e}-2$ & 0.4 & $2.2 \mathrm{e}-3$ & 1.0 & $2.2 \mathrm{e}-1$ & 0.1 & $1.1 \mathrm{e}-1$ & 0.5
\end{tabular}

TABLE 4. $L^{\infty}$ - and $L^{1}$-errors with $\mathrm{MpR}$ at $T=1$ with $I=[-100,100]$ for (5.1) with $\varphi$ given by (5.4) and $g$ by (5.2) with $v(x, t)=(t+1) \mathrm{e}^{-|x|^{2}}$.

5.4. Fractional Fast Diffusion Equation. In the fast diffusion case a new difficulty appears: The nonlinearity, $\varphi(\xi)=\xi^{m}$ for $m \in(0,1)$, is no longer locally Lipschitz, and the (CFL) condition can only hold for implicit schemes or under approximation of $\varphi$. Even in the local case there are few results for this case, and the results in this paper are as far as we know the first in the fractional case.

5.4.1. Implicit scheme. Implicit schemes automatically satisfy (CFL) at the price of having to solve a nonlinear system of equations at every time step. This is computationally very expensive. For simplicity we use the standard nonlinear solver "fsolve" in Matlab. A more adapted solver could probably significantly reduce the computational time. Due to the computational cost, we take a very small domain and a very regular solution. We consider (5.1) with $\varphi(\xi)=\xi^{\frac{1}{2}}, \alpha=1$ and $g$ given by (5.2) with $v(x, t)=\sqrt{(t+1)} \mathrm{e}^{-|x|^{8}}$ (the solution). We run the experiments with the Midpoint Rule up to time $T=1$ in the domain $I=[-4,4]$. For the time discretization we choose $\Delta t \sim h$ and $\Delta t \sim h^{2}$. We also consider the Crank-Nicolson 


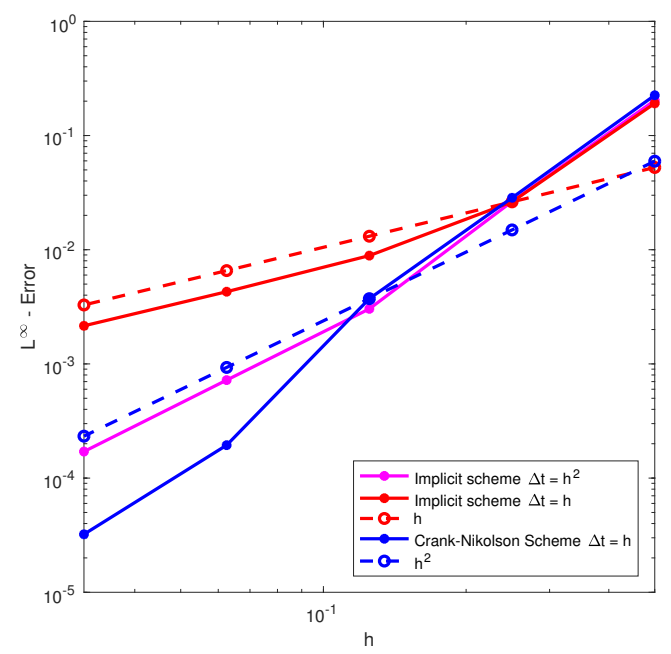

FIGURE 5. $L^{\infty}$-error with $\mathrm{MpR}$ at $T=1$ with $I=[-4,4]$ for (5.1) with $\varphi(\xi)=\xi^{\frac{1}{2}}, \alpha=1$ and $g$ given by (5.2) with $v(x, t)=\sqrt{(t+1)} \mathrm{e}^{-|x|^{8}}$.

\begin{tabular}{|c|c|c|c|c|c|c|}
\hline \multirow[t]{2}{*}{$h$} & Implicit & $\Delta t \sim h$ & Implicit & $\Delta t \sim h^{2}$ & $\mathrm{Cr}-\mathrm{Ni}$ & $\Delta t \sim h$ \\
\hline & error & $\gamma$ & error & $\gamma$ & error & $\gamma$ \\
\hline $5.00 \mathrm{e}-1$ & $1.91 \mathrm{e}-1$ & & $2.03 \mathrm{e}-1$ & & $2.25 \mathrm{e}-1$ & \\
\hline $2.50 \mathrm{e}-1$ & $2.63 \mathrm{e}-2$ & 2.86 & $2.64 \mathrm{e}-2$ & 2.95 & $2.85 \mathrm{e}-2$ & 2.98 \\
\hline $1.25 \mathrm{e}-1$ & $8.88 \mathrm{e}-3$ & 1.56 & $3.03 \mathrm{e}-3$ & 3.12 & $3.73 \mathrm{e}-3$ & 2.93 \\
\hline $6.25 \mathrm{e}-2$ & $4.29 \mathrm{e}-3$ & 1.05 & $7.21 \mathrm{e}-4$ & 2.07 & $1.94 \mathrm{e}-4$ & 4.26 \\
\hline $3.13 \mathrm{e}-2$ & $2.16 \mathrm{e}-3$ & 1.00 & $1.71 \mathrm{e}-4$ & 2.08 & $3.22 \mathrm{e}-5$ & 2.59 \\
\hline
\end{tabular}

TABLE 5. $L^{\infty}$-error with $\mathrm{MpR}$ at $T=1$ with $I=[-4,4]$ for $(5.1)$ with $\varphi(\xi)=\xi^{\frac{1}{2}}, \alpha=1$ and $g$ given by $(5.2)$ with $v(x, t)=\sqrt{(t+1)} \mathrm{e}^{-|x|^{8}}$.

method $\left(\theta=\frac{1}{2}\right)$ with $\Delta t \sim h$ which means that (CFL) is satisfied. The results are shown in Figure 5 and Table 5 (above).

Conclusion: For the implicit method with the Midpoint Rule (a second order method when $\alpha=1)$, the expected error is $O\left(h^{2}+\Delta t\right)$. When $\Delta t \sim h$ the error is clearly governed by $\Delta t$ and so the rate of convergence is linear. For $\Delta t \sim h^{2}$, the error introduced by the time discretization is proportional to $h^{2}$ and so the rate of convergence is quadratic. For the Crank-Nicolson method, the expected error is $O\left(h^{2}+\Delta t^{2}\right)$, so with $\Delta t \sim h$ we should see second order convergence. However the observed rates are better, see Table 5 . The Crank-Nicolson methods seems to be better than both implicit methods in terms of computational time and accuracy.

5.4.2. Explicit scheme approximating the nonlinearity. We consider (5.1) with $g \equiv$ $0, \varphi(\xi)=\xi^{m}, m=0.6$, and $\alpha=1.5$, and note that by [49] the solution is given by

$$
v(x, t)=\lambda(t+1)^{-\beta}\left(1+\left(|x|(t+1)^{-\beta}\right)^{2}\right)^{-\frac{\alpha+1}{2}}
$$

for $\beta=\frac{1}{m-1+\alpha}$ and $\lambda=\left(\frac{2^{\alpha-1}}{\beta} \frac{\Gamma((1+\alpha) / 2)}{\Gamma((3-\alpha) / 2)}\right)^{1 /(1-m)}$. We use the approach of Section 4.6 and implement an explicit method with the Second Order Interpolation + Adapted Vanishing Viscosity (SOI) + approximate nonlinearity $\varphi^{\epsilon}(\xi)=(\xi+$ $\epsilon)^{0.6}-\epsilon^{0.6}$. We run experiments for up to time $T=1$ with $I=[-1000,1000]$ for $\Delta t$ 's satisfying $(\mathrm{CFL})$ for $\varphi^{\epsilon}$. The results are shown in Figure 6 (above) and Table 6 (below). 


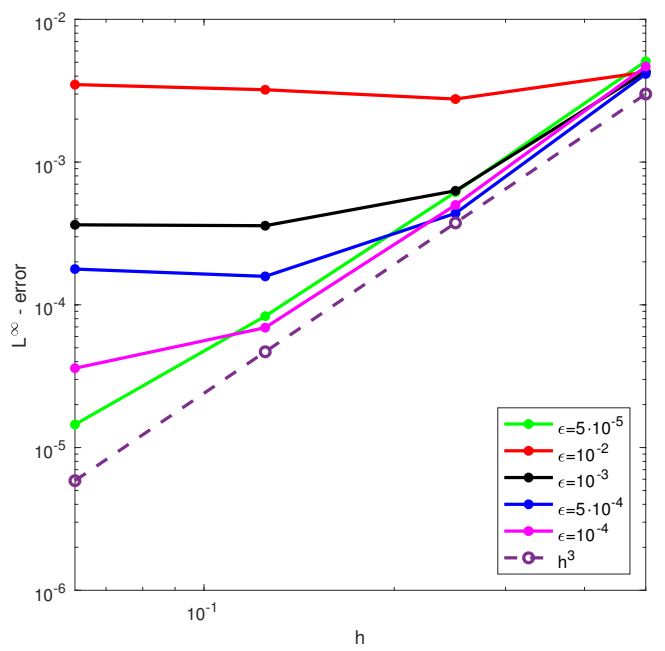

FIGURE $6 . L^{\infty}$-errors with SOI at $T=1$ with $I=[-1000,1000]$ for the exact solution (5.5) of (5.1) with $g \equiv 0, \varphi(\xi)=\xi^{0.6}$ and $\alpha=1.5$, approximating the nonlinearity by $\varphi^{\epsilon}(\xi)=(\xi+\epsilon)^{0.6}-\epsilon^{0.6}$.

\begin{tabular}{l|l|l|l|l|l|l|l|l|l}
\multicolumn{2}{|c|}{$h$} & \multicolumn{3}{|c|}{$\epsilon=5 \mathrm{e}-4$} & \multicolumn{3}{c|}{$\epsilon=1 \mathrm{e}-4$} & \multicolumn{3}{c}{$\epsilon=5 \mathrm{e}-5$} \\
\cline { 2 - 10 } & $\Delta t$ & error & $\gamma$ & $\Delta t$ & error & $\gamma$ & $\Delta t$ & error & $\gamma$ \\
\hline $5.00 \mathrm{e}-1$ & $9.39 \mathrm{e}-3$ & $4.14 \mathrm{e}-3$ & & $4.93 \mathrm{e}-3$ & $4.67 \mathrm{e}-3$ & & $1.97 \mathrm{e}-3$ & $5.09 \mathrm{e}-3$ & \\
$2.50 \mathrm{e}-1$ & $3.32 \mathrm{e}-3$ & $4.38 \mathrm{e}-4$ & 3.24 & $1.74 \mathrm{e}-3$ & $5.00 \mathrm{e}-4$ & 3.22 & $6.16 \mathrm{e}-4$ & $6.16 \mathrm{e}-4$ & 3.06 \\
$1.25 \mathrm{e}-1$ & $1.17 \mathrm{e}-3$ & $1.58 \mathrm{e}-4$ & 1.47 & $6.17 \mathrm{e}-4$ & $6.92 \mathrm{e}-5$ & 2.85 & $8.32 \mathrm{e}-5$ & $8.32 \mathrm{e}-5$ & 2.89 \\
$6.25 \mathrm{e}-2$ & $4.15 \mathrm{e}-4$ & $1.78 \mathrm{e}-4$ & -0.17 & $2.18 \mathrm{e}-4$ & $3.60 \mathrm{e}-5$ & 0.94 & $1.44 \mathrm{e}-5$ & $1.45 \mathrm{e}-5$ & 2.52
\end{tabular}

TABLE $6 . L^{\infty}$-errors with SOI at $T=1$ with $I=[-1000,1000]$ for the exact solution (5.5) of (5.1) with $f \equiv 0, \varphi(\xi)=\xi^{0.6}$ and $\alpha=1.5$, approximating the nonlinearity by $\varphi^{\epsilon}(\xi)=(\xi+\epsilon)^{0.6}-\epsilon^{0.6}$. Note that we have only included the most accurate approximations here.

Conclusion: Here the expected error is $O\left(h^{3-\alpha}+\Delta t\right)$ or $O\left(h^{1.5}\right)$ when $\alpha=1.5$ and $\Delta t \sim h^{\alpha}$. Since we are approximating the nonlinearity, the (CFL) condition becomes more and more restrictive when $\epsilon$ is decreased. When we fix $\epsilon$ and let $h, \Delta t \rightarrow 0^{+}$, the error stops decreasing at some point as can be seen in Figure 6 and Table 6. However, very good results are obtained for small $\epsilon$. Compared with the implicit method of the previous section, the present method is better both in terms of computational times (we are able to deal with much bigger domains) and accuracy (we reach almost $10^{-6}$ with $h=6.25 \mathrm{e}-2$ instead of $h=3.13 \mathrm{e}-2$ ).

5.5. On the truncation of the domain. To test numerically the effect of the restriction to a large bounded domain, we consider the Fractional Heat Equation and the explicit solution of Section 5.1. An explicit PDL scheme is run up to $T=1$ on a sequence of increasing domains. We take $\Delta t \sim h^{2}$ which satisfies (CFL) and ensures that the space discretization errors dominate. See Figure 7 for the results. We also test the minimal error that can be reached for a fixed domain for different values of $\alpha$. Here we consider the Fractional Porous Medium Equation with $m=(3-\alpha) /(1+\alpha)$ and explicit solutions from [49]. See Figure 8.

Conclusion: The expected error for our schemes are $O\left(h^{2}+\Delta t\right)=O\left(h^{2}\right)$ since $\Delta t \sim h^{2}$. For each fixed domain $I$, we see from Figure 7 that the errors decrease as $h \rightarrow 0^{+}$down to some threshold below which there is no improvement. At these thresholds, the dominant error comes from the truncation of the domain. As 

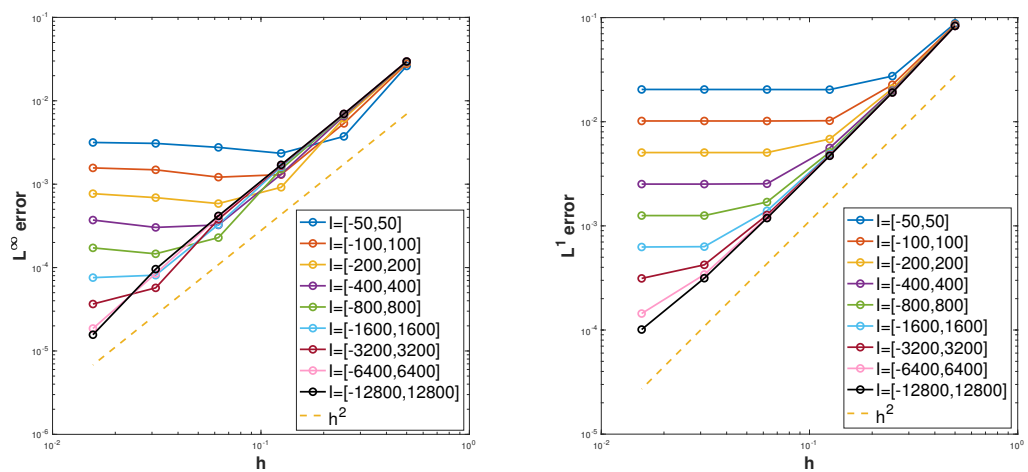

FIGURE 7. $L^{\infty}$ - and $L^{1}$-errors with PDL at $T=1$ with different interval sizes $I$ for the exact solution (5.3) of (5.1) with $\alpha=1, \varphi(\xi)=\xi$, and $g \equiv 0$.

expected, these thresholds decrease as the size of the domain increases. Figure 8 shows that when you vary $\alpha$, the minimal reachable errors for a fixed domain $I$ is of the order of length $(I)^{-\alpha}$. This could be an indication that the error due to the truncation of the domain is determined by the tail behaviour of the Lévy measure - here $\mathrm{d} \mu(z) \sim|z|^{-1-\alpha} \mathrm{d} z$. Such behaviour would be consistent with the analytical results of [9] for tempered Lévy models (which do not include the fractional Laplacian). Another analytical approach using Barenblatt solutions (cf. [68]) can be found in [33].

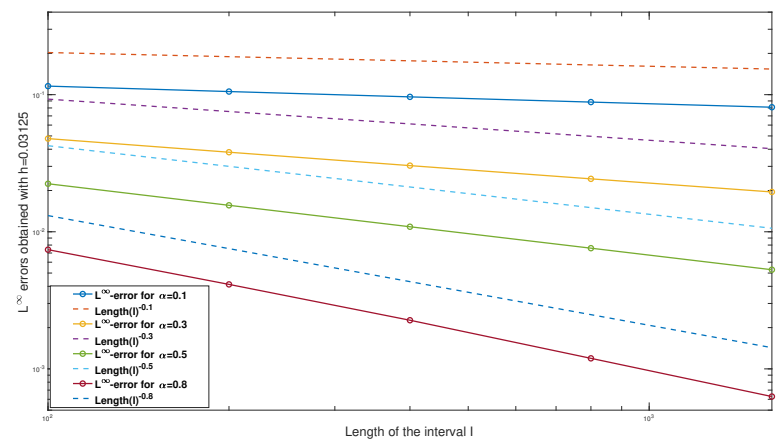

FiguRE 8. Minimal $L^{\infty}$ - and $L^{1}$-errors with PDL at $T=1$ with different interval sizes $I$ for the exact solution of (5.1) with $\varphi(\xi)=\xi^{\frac{3}{1+\alpha}}$, and $g \equiv 0$.

\section{NUMERICAL EXPERIMENTS IN 2D}

In this section we test our numerical schemes on more complicated problems in two space dimensions which has a much richer solution structure and a more interesting evolution. We consider Stefan problems of the type

$$
\partial_{t} u(x, y, t)+\mathfrak{L}_{i}^{\sigma, \mu}[\varphi(u(\cdot, \cdot, t))](x, y)=0 \quad \text { in } \quad Q_{T}:=\mathbb{R}^{2} \times(0, T)
$$


for $i=1,2, \varphi(\xi)=\max \{0, \xi-1\}$, and both $x$-directed nonlocal diffusion and "diagonal" $\vec{\sigma}^{T}=\left(\frac{1}{2}, \frac{47}{100}\right)$-directed local diffusion,

$$
\begin{aligned}
& \mathfrak{L}_{1}^{\sigma, \mu}[\psi](x, y):=(\vec{\sigma} \cdot D)^{2}[\psi](x, y)+\left(-\partial_{x x}\right)^{\frac{1}{4}}[\psi](x, y), \\
& \mathfrak{L}_{2}^{\sigma, \mu}[\psi](x, y):=(\vec{\sigma} \cdot D)^{2}[\psi](x, y)+\frac{1}{10}\left(-\partial_{x x}\right)^{\frac{1}{4}}[\psi](x, y) .
\end{aligned}
$$

Note that nonlocal diffusion is stronger in $\mathfrak{L}_{1}^{\sigma, \mu}$ than in $\mathfrak{L}_{2}^{\sigma, \mu}$.

As in Section 5, we use an equidistant grid in space and time and restrict to a (large) bounded spatial domain $I_{1} \times I_{2} \subset \mathbb{R}^{2}$ (setting the numerical solution equal zero outside). The errors are computed numerically under the same assumptions as before. We run an explicit scheme $(\theta=1)$ with $\Delta t \sim h^{2}$ such that (CFL) holds and the overall order of convergence is determined by the spatial discretization. Since $x+\vec{\sigma} \eta$ is not aligned with the spatial grid $\mathcal{G}_{h}$, we discretize the local term by (4.5) and $\eta=\sqrt{h}$ which leads to $O(h)$ errors (cf. Lemma 4.2). For the nonlocal diffusion, we use the 1-dimensional version of Powers of the Discrete Laplacian (PDL) of Section 4.5 which has $O\left(h^{2}\right)$ errors. We also choose a rough initial data (see Figure 10 and Figure 11) given by

$$
u_{0}(x, y)=3\left(\mathbf{1}_{S_{1}}(x, y)-\mathbf{1}_{S_{2}}(x, y)\right)+4 \mathbf{1}_{S_{3}}(x, y)
$$

where $S_{1}=\left\{(x, y) \in \mathbb{R}^{2}:|x|<5 \&|y|<5\right\}, S_{2}=\left\{(x, y) \in \mathbb{R}^{2}:|x|<2 \&|y|<2\right\}$ and $S_{3}=\left\{(x, y) \in \mathbb{R}^{2}: 3<|x|<4 \& 3<|y|<4\right\}$. We run the experiments on the domain $I_{1} \times I_{2}=[-100,100] \times[-10,10]$. The different sizes of the domain in the $x$ - and $y$-directions are adapted to the combination of compactly supported data and degenerate operators (6.2) and (6.3). These operators are nonlocal in the $x$-direction which requires a wide domain there. The size of the domain in the $y$-direction can be smaller because the Stefan problem with local diffusion has finite speed of propagation (see e.g. the introduction of [10]).

In figure 9 we list the relative $L^{\infty}$ - and $L^{1}$-errors (errors divided by the $L^{\infty}$ and $L^{1}$-norms, respectively, of the solution) for the numerical solution of (6.1) with $i=1$. In Figure 10 (resp. Figure 11) we plot, for different times, the numerical solution of (6.1) with $i=1$ (resp. $i=2$ ).

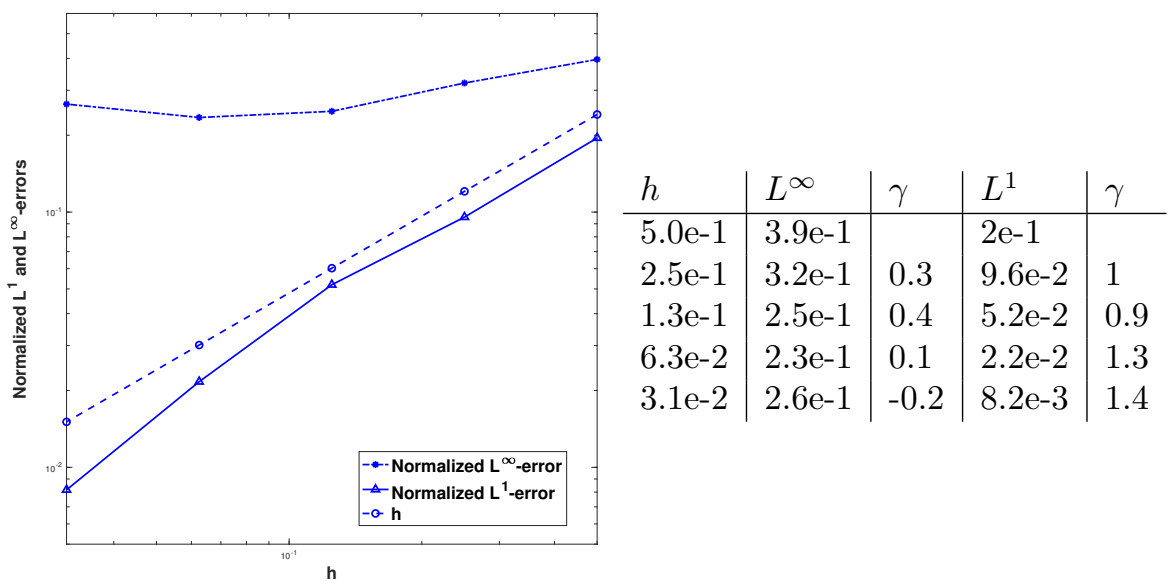

FiguRE 9. Relative $L^{\infty}$ - and $L^{1}$-errors with (4.5) and PDL discretizations at $T=1$ with $I_{1} \times I_{2}=[-100,100] \times[-10,10]$ for $(6.1)$ with $\varphi(\xi)=\max \{0, \xi-$ $1\}$ and the diffusion operator given by (6.2).

Conclusion: Figure 9 confirms the $O(h)$ convergence in $L^{1}$ predicted by Lemma 4.2. As in Section 5.3, there is no convergence in $L^{\infty}$. In Figure 10 there are 

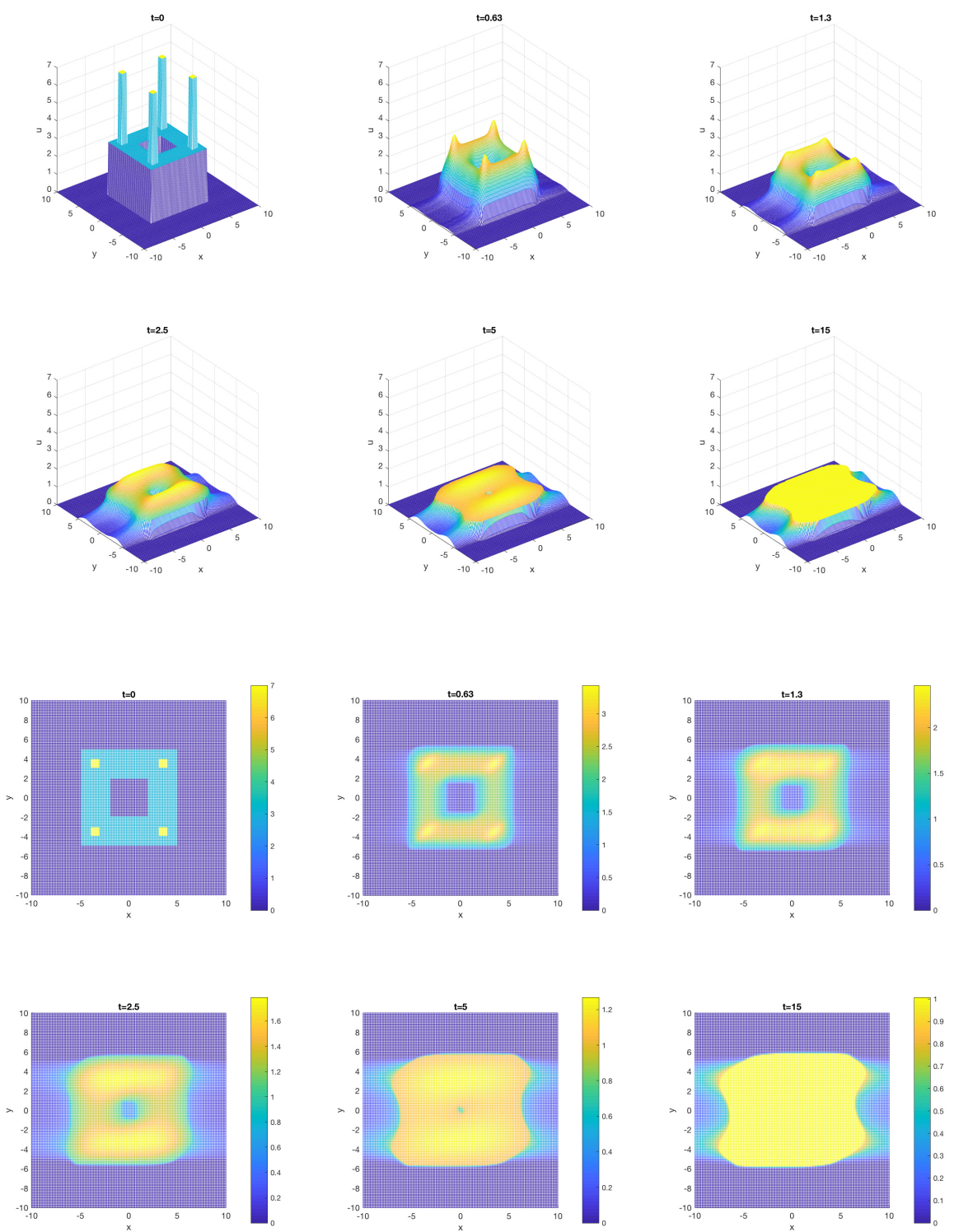

FIGURE 10. Stronger nonlocal diffusion. Solution of (6.1) with $\varphi(\xi)=$ $\max \{0, \xi-1\}$ and the diffusion operator given by (6.2).

pronounced long "bands" due to the nonlocal diffusion, and the effect of the almost diagonal local diffusion is also visible. In Figure 11 the nonlocal diffusion is weaker and the local diffusion dominates. The "long bands" are not very pronounced. Both figures exhibit regions of discontinuity of the solution in the $y$-direction (where there is only local diffusion). Such behaviour is well-known for local Stefan problems.

\section{ACKNOWLEDGEMENTS}

All authors were supported by the Toppforsk (research excellence) project Waves and Nonlinear Phenomena (WaNP), grant no. 250070 from the Research Council of Norway. F. del Teso was also supported by the BERC 2018-2021 program from the Basque Government, BCAM Severo Ochoa excellence accreditation SEV-2017-0718 

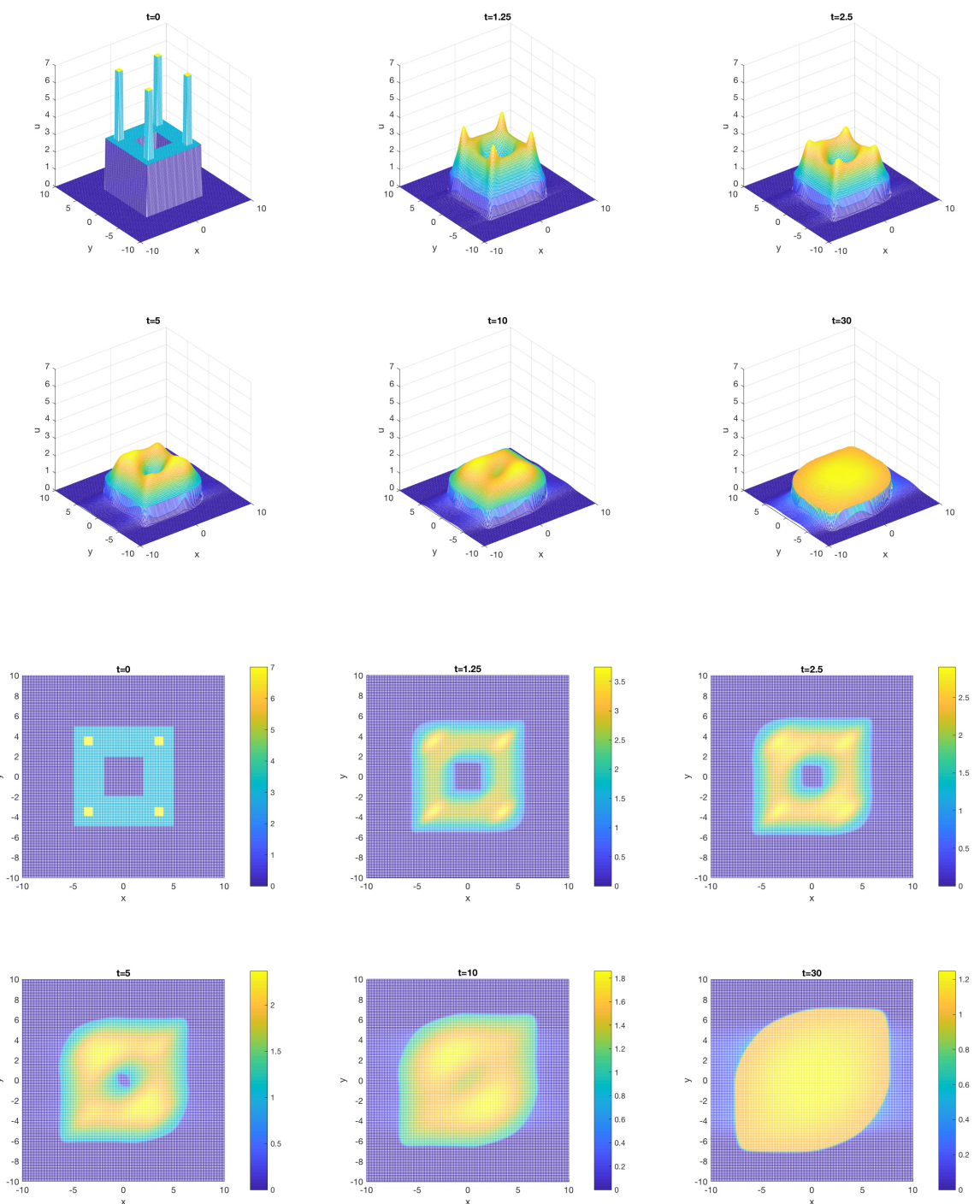

FIGURE 11. Weak nonlocal diffusion. Solution of (6.1) with $\varphi(\xi)=$ $\max \{0, \xi-1\}$ and the diffusion operator given by (6.3).

from Spanish Ministry of Economy and Competitiveness (MINECO), the ERCIM "Alain Bensoussan" Fellowship programme and the "Juan de la Cierva - formación" program (FJCI-2016-30148). We would like to thank the anonymous referees for helping us improve the paper and Indranil Chowdhury for a careful reading and useful comments.

\section{REFERENCES}

[1] N. Alibaud and B. Andreianov. Non-uniqueness of weak solutions for the fractal Burgers equation. Ann. Inst. H. Poincaré Anal. Non Linéaire, 27(4):9971016, 2010.

[2] N. Alibaud, S. Cifani, and E. R. Jakobsen. Optimal continuous dependence estimates for fractional degenerate parabolic equations. Arch. Ration. Mech. Anal., 213(3):705-762, 2014. 
[3] F. Andreu-Vaillo, J. M. Mazón, J. D. Rossi, and J. J. Toledo-Melero. Nonlocal diffusion problems, volume 165 of Mathematical Surveys and Monographs. American Mathematical Society, Providence, RI; Real Sociedad Matemática Española, Madrid, 2010.

[4] D. Applebaum. Lévy processes and stochastic calculus, volume 116 of Cambridge Studies in Advanced Mathematics. Cambridge University Press, Cambridge, second edition, 2009.

[5] S. Asmussen and J. Rosiński. Approximations of small jumps of Lévy processes with a view towards simulation. J. Appl. Probab., 38(2):482-493, 2001.

[6] A. Benedek and R. Panzone. The spaces $L^{p}$, with mixed norm. Duke Math. J., 28:301-324, 1961.

[7] J. Bertoin. Lévy processes, volume 121 of Cambridge Tracts in Mathematics. Cambridge University Press, Cambridge, 1996.

[8] I. H. Biswas, E. R. Jakobsen, and K. H. Karlsen. Difference-quadrature schemes for nonlinear degenerate parabolic integro-PDE. SIAM J. Numer. Anal., 48(3):1110-1135, 2010.

[9] C. Brändle and E. Chasseigne. Large deviation estimates for some nonlocal equations. General bounds and applications. Trans. Amer. Math. Soc., 365(7):3437-3476, 2013.

[10] C. Brändle, E. Chasseigne, and F. Quirós. Phase transitions with midrange interactions: a nonlocal Stefan model. SIAM J. Math. Anal., 44(4):3071-3100, 2012.

[11] H. Brézis and M. G. Crandall. Uniqueness of solutions of the initial-value problem for $u_{t}-\Delta \varphi(u)=0$. J. Math. Pures Appl. (9), 58(2):153-163, 1979.

[12] C. Bucur and E. Valdinoci. Nonlocal diffusion and applications, volume 20 of Lecture Notes of the Unione Matematica Italiana. Springer, [Cham]; Unione Matematica Italiana, Bologna, 2016.

[13] R. Bürger, A. Coronel, and M. Sepúlveda. A semi-implicit monotone difference scheme for an initial-boundary value problem of a strongly degenerate parabolic equation modeling sedimentation-consolidation processes. Math. Comp., 75(253):91-112, 2006.

[14] L. Caffarelli. Non-local diffusions, drifts and games. In Nonlinear partial differential equations, volume 7 of Abel Symp., pages 37-52. Springer, Heidelberg, 2012.

[15] L. Caffarelli and L. Silvestre. An extension problem related to the fractional Laplacian. Comm. Partial Differential Equations, 32(7-9):1245-1260, 2007.

[16] F. Camilli and M. Falcone. An approximation scheme for the optimal control of diffusion processes. RAIRO Modél. Math. Anal. Numér., 29(1):97-122, 1995.

[17] F. Camilli and E. R. Jakobsen. A finite element like scheme for integropartial differential Hamilton-Jacobi-Bellman equations. SIAM J. Numer. Anal., 47(4):2407-2431, 2009.

[18] C. H. Chan, M. Czubak, and L. Silvestre. Eventual regularization of the slightly supercritical fractional Burgers equation. Discrete Contin. Dyn. Syst., 27(2):847-861, 2010.

[19] P. G. Ciarlet and P.-A. Raviart. General Lagrange and Hermite interpolation in $\mathbf{R}^{n}$ with applications to finite element methods. Arch. Rational Mech. Anal., 46:177-199, 1972.

[20] O. Ciaurri, L. Roncal, P. R. Stinga, J. L. Torrea, and J. L. Varona. Fractional discrete laplacian versus discretized fractional laplacian. Preprint, arXiv:1507.04986v1 [math.AP], 2015.

[21] O. Ciaurri, L. Roncal, P. R. Stinga, J. L. Torrea, and J. L. Varona. Nonlocal discrete diffusion equations and the fractional discrete Laplacian, regularity 
and applications. Adv. Math., 330:688-738, 2018.

[22] S. Cifani and E. R. Jakobsen. Entropy solution theory for fractional degenerate convection-diffusion equations. Ann. Inst. H. Poincaré Anal. Non Linéaire, 28(3):413-441, 2011.

[23] S. Cifani and E. R. Jakobsen. On the spectral vanishing viscosity method for periodic fractional conservation laws. Math. Comp., 82(283):1489-1514, 2013.

[24] S. Cifani and E. R. Jakobsen. On numerical methods and error estimates for degenerate fractional convection-diffusion equations. Numer. Math., 127(3):447483, 2014.

[25] S. Cifani, E. R. Jakobsen, and K. H. Karlsen. The discontinuous Galerkin method for fractional degenerate convection-diffusion equations. BIT, 51(4):809-844, 2011.

[26] R. Cont and P. Tankov. Financial modelling with jump processes. Chapman \& Hall/CRC Financial Mathematics Series. Chapman \& Hall/CRC, Boca Raton, FL, 2004 .

[27] N. Cusimano, F. del Teso, L. Gerardo-Giorda, and G. Pagnini. Monotone discretizations of the fractional Laplacian in $\mathbb{R}^{n}$ trough the heat semigroup formula. In preparation, 2018.

[28] N. Cusimano, F. del Teso, L. Gerardo-Giorda, and G. Pagnini. Discretizations of the spectral fractional Laplacian on general domains with Dirichlet, Neumann, and Robin boundary conditions. SIAM J. Numer. Anal., 56(3):12431272, 2018.

[29] A. de Pablo, F. Quirós, and A. Rodríguez. Nonlocal filtration equations with rough kernels. Nonlinear Anal., 137:402-425, 2016.

[30] A. de Pablo, F. Quirós, A. Rodríguez, and J. L. Vázquez. A fractional porous medium equation. Adv. Math., 226(2):1378-1409, 2011.

[31] A. de Pablo, F. Quirós, A. Rodríguez, and J. L. Vázquez. A general fractional porous medium equation. Comm. Pure Appl. Math., 65(9):1242-1284, 2012.

[32] K. Debrabant and E. R. Jakobsen. Semi-Lagrangian schemes for parabolic equations. In Recent developments in computational finance, volume 14 of Interdiscip. Math. Sci., pages 279-297. World Sci. Publ., Hackensack, NJ, 2013.

[33] F. del Teso. Finite difference method for a fractional porous medium equation. Calcolo, 51(4):615-638, 2014.

[34] F. del Teso, J. Endal, and E. R. Jakobsen. On distributional solutions of local and nonlocal problems of porous medium type. C. R. Math. Acad. Sci. Paris, 355(11):1154-1160, 2017.

[35] F. del Teso, J. Endal, and E. R. Jakobsen. Uniqueness and properties of distributional solutions of nonlocal equations of porous medium type. Adv. Math., 305:78-143, 2017.

[36] F. del Teso, J. Endal, and E. R. Jakobsen. Robust numerical methods for nonlocal (and local) equations of porous medium type. Part I: Theory. Submitted, preprint available at arXiv, arXiv:1801.07148v1 [math.NA], 2018.

[37] F. del Teso and J. L. Vázquez. Finite difference method for a general fractional porous medium equation. Preprint, arXiv:1307.2474v1 [math.NA], 2014.

[38] E. DiBenedetto and D. Hoff. An interface tracking algorithm for the porous medium equation. Trans. Amer. Math. Soc., 284(2):463-500, 1984.

[39] J. Droniou. A numerical method for fractal conservation laws. Math. Comp., 79(269):95-124, 2010.

[40] J. Droniou, R. Eymard, T. Gallouet, and R. Herbin. Gradient schemes: a generic framework for the discretisation of linear, nonlinear and nonlocal elliptic and parabolic equations. Math. Models Methods Appl. Sci., 23(13):23952432, 2013. 
[41] J. Droniou, R. Eymard, and R. Herbin. Gradient schemes: generic tools for the numerical analysis of diffusion equations. ESAIM Math. Model. Numer. Anal., 50(3):749-781, 2016.

[42] J. Droniou and C. Imbert. Fractal first-order partial differential equations. Arch. Ration. Mech. Anal., 182(2):299-331, 2006.

[43] J. Droniou and E. R. Jakobsen. A uniformly converging scheme for fractal conservation laws. In Finite volumes for complex applications VII. Methods and theoretical aspects, volume 77 of Springer Proc. Math. Stat., pages 237245. Springer, Cham, 2014.

[44] J. C. M. Duque, R. M. P. Almeida, and S. N. Antontsev. Convergence of the finite element method for the porous media equation with variable exponent. SIAM J. Numer. Anal., 51(6):3483-3504, 2013.

[45] C. Ebmeyer and W. B. Liu. Finite element approximation of the fast diffusion and the porous medium equations. SIAM J. Numer. Anal., 46(5):2393-2410, 2008.

[46] E. Emmrich and D. Šiška. Full discretization of the porous medium/fast diffusion equation based on its very weak formulation. Commun. Math. Sci., 10(4):1055-1080, 2012.

[47] S. Evje and K. H. Karlsen. Monotone difference approximations of BV solutions to degenerate convection-diffusion equations. SIAM J. Numer. Anal., 37(6):1838-1860, 2000.

[48] R. Eymard, T. Gallouët, and R. Herbin. Finite volume methods. In Handbook of numerical analysis, Vol. VII, Handb. Numer. Anal., VII, pages 713-1020. North-Holland, Amsterdam, 2000.

[49] Y. Huang. Explicit Barenblatt profiles for fractional porous medium equations. Bull. Lond. Math. Soc., 46(4):857-869, 2014.

[50] Y. Huang and A. Oberman. Numerical methods for the fractional Laplacian: a finite difference-quadrature approach. SIAM J. Numer. Anal., 52(6):30563084, 2014.

[51] Y. Huang and A. Oberman. Finite difference methods for fractional Laplacians. Preprint, arXiv:1611.00164v1 [math.NA], 2016.

[52] L. Ignat and D. Stan. Asymptotic behaviour of solutions to fractional diffusionconvection equations. J. Lond. Math. Soc. (2), 97(2):258-281, 2018.

[53] E. R. Jakobsen, K. H. Karlsen, and C. La Chioma. Error estimates for approximate solutions to Bellman equations associated with controlled jumpdiffusions. Numer. Math., 110(2):221-255, 2008.

[54] S. Jerez and C. Parés. Entropy stable schemes for degenerate convectiondiffusion equations. SIAM J. Numer. Anal., 55(1):240-264, 2017.

[55] K. H. Karlsen, N. H. Risebro, and E. B. Storrøsten. On the convergence rate of finite difference methods for degenerate convection-diffusion equations in several space dimensions. ESAIM Math. Model. Numer. Anal., 50(2):499-539, 2016.

[56] C. Lizama and L. Roncal. Hölder-Lebesgue regularity and almost periodicity for semidiscrete equations with a fractional Laplacian. Discrete Continuous Dyn. Syst., 38(3):1365-1403, 2018.

[57] R. Metzler and J. Klafter. The restaurant at the end of the random walk: recent developments in the description of anomalous transport by fractional dynamics. J. Phys. A, 37(31):R161-R208, 2004.

[58] A. Mizutani, N. Saito, and T. Suzuki. Finite element approximation for degenerate parabolic equations. An application of nonlinear semigroup theory. M2AN Math. Model. Numer. Anal., 39(4):755-780, 2005. 
[59] L. Monsaingeon. An explicit finite-difference scheme for one-dimensional generalized porous medium equations: interface tracking and the hole filling problem. ESAIM Math. Model. Numer. Anal., 50(4):1011-1033, 2016.

[60] R. H. Nochetto, E. Otárola, and A. J. Salgado. A PDE approach to fractional diffusion in general domains: a priori error analysis. Found. Comput. Math., 15(3):733-791, 2015.

[61] R. H. Nochetto and C. Verdi. Approximation of degenerate parabolic problems using numerical integration. SIAM J. Numer. Anal., 25(4):784-814, 1988.

[62] M. E. Rose. Numerical methods for flows through porous media. I. Math. Comp., 40(162):435-467, 1983.

[63] J. Rulla and N. J. Walkington. Optimal rates of convergence for degenerate parabolic problems in two dimensions. SIAM J. Numer. Anal., 33(1):56-67, 1996.

[64] W. Schoutens. Lévy Processes in Finance: Pricing Financial Derivatives. Wiley series in probability and statistics. Wiley, Chichester, first edition, 2003.

[65] E. Valdinoci. From the long jump random walk to the fractional Laplacian. Bol. Soc. Esp. Mat. Apl. S $\overrightarrow{\mathrm{e} M A, ~ 49: 33-44, ~} 2009$.

[66] J. L. Vázquez. The porous medium equation. Mathematical theory. Oxford Mathematical Monographs. The Clarendon Press, Oxford University Press, Oxford, 2007.

[67] J. L. Vázquez. Nonlinear diffusion with fractional Laplacian operators. In Nonlinear partial differential equations, volume 7 of Abel Symp., pages 271298. Springer, Heidelberg, 2012.

[68] J. L. Vázquez.ut Barenblatt solutions and asymptotic behaviour for a nonlinear fractional heat equation of porous medium type. J. Eur. Math. Soc. (JEMS), 16(4):769-803, 2014.

[69] J. L. Vázquez, A. de Pablo, F. Quirós, and A. Rodríguez. Classical solutions and higher regularity for nonlinear fractional diffusion equations. J. Eur. Math. Soc. (JEMS), 19(7):1949-1975, 2017.

[70] W. A. Woyczyński. Lévy processes in the physical sciences. In Lévy processes, pages 241-266. Birkhäuser Boston, Boston, MA, 2001.

[71] Q. Xu and J. S. Hesthaven. Discontinuous Galerkin method for fractional convection-diffusion equations. SIAM J. Numer. Anal., 52(1):405-423, 2014.

[72] Q. Zhang and Z.-L. Wu. Numerical simulation for porous medium equation by local discontinuous Galerkin finite element method. J. Sci. Comput., 38(2):127-148, 2009.

(F. del Teso) Basque Center for Applied Mathematics (BCAM), Bilbao, Spain

Email address: fdelteso@bcamath.org

$U R L:$ http://www.bcamath.org/es/people/fdelteso

(J. Endal) Department of Mathematical Sciences, Norwegian University of Science And Technology (NTNU), N-7491 Trondheim, Norway

Email address: jorgen.endal@ntnu.no

$U R L:$ http://folk.ntnu.no/jorgeen

(E. R. Jakobsen) Department of Mathematical Sciences, Norwegian University of Science and Technology (NTNU), N-7491 Trondheim, Norway

Email address: espen.jakobsen@ntnu.no

URL: http://www.math.ntnu.no/ ${ }^{\text {erj } /}$ 